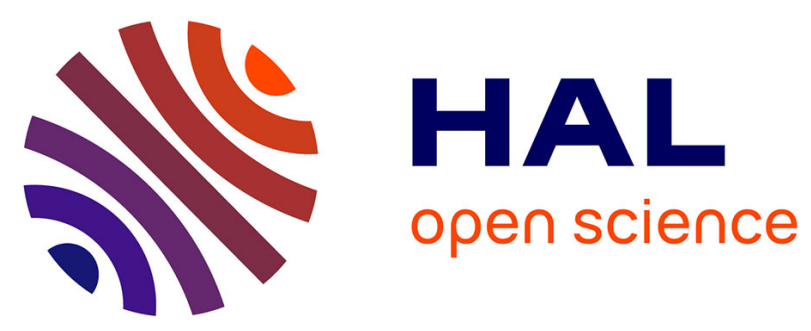

\title{
Development of a joint refinement model for the spin-resolved one-electron reduced density matrix using different data sets
}

\author{
Saber Gueddida, Zeyin Yan, Jean-Michel Gillet
}

\section{- To cite this version:}

Saber Gueddida, Zeyin Yan, Jean-Michel Gillet. Development of a joint refinement model for the spinresolved one-electron reduced density matrix using different data sets. Acta Crystallographica Section A : Foundations and Advances [2014-..], 2018, 74 (2), pp.131 - 142. 10.1107/S2053273318000384. hal-01795046

\author{
HAL Id: hal-01795046 \\ https://hal.science/hal-01795046
}

Submitted on 25 Sep 2020

HAL is a multi-disciplinary open access archive for the deposit and dissemination of scientific research documents, whether they are published or not. The documents may come from teaching and research institutions in France or abroad, or from public or private research centers.
L'archive ouverte pluridisciplinaire HAL, est destinée au dépôt et à la diffusion de documents scientifiques de niveau recherche, publiés ou non, émanant des établissements d'enseignement et de recherche français ou étrangers, des laboratoires publics ou privés. 


\title{
Development of a joint refinement model for the spin resolved one-electron-reduced density matrix using different data sets
}

\author{
Saber Gueddida, ${ }^{1}$ Zeyin Yan, ${ }^{1}$ and Jean-Michel Gillet ${ }^{1}$ \\ ${ }^{1}$ SPMS, UMR8580, CentraleSupelec, Université Paris-Saclay, \\ 3 rue Joliot-Curie, Gif-sur-Yvette 91190, France.
}

\begin{abstract}
The paper describes a joint refinement model of the spin resolved one-electron-reduced density matrix using simultaneously magnetic structure factors and magnetic directional Compton profiles. The model is guided by two strategies: (i) variation of basis functions and (ii) variation of the spin population matrix. Our implementation for a finite system is based on an expansion of the natural orbitals on basis sets. To show the potential benefits brought by the joint refinement model, we also present the refinement results using magnetic structure factors only. The joint refinement model provides very satisfactory results reproducing the pseudo data. In particular, magnetic Compton profiles have a strong effect not only on the off-diagonal elements of the spin resolved one-electron-reduced density matrix but also on its diagonal elements.
\end{abstract}




\section{INTRODUCTION}

Reduced density matrix is an alternative way of describing a system of $N$ interacting particles compared with the $N$-electron wavefunction ${ }^{12}$. The one-electron-reduced density matrix $(1-R D M)^{3-6}$ is widely accepted as playing a central role in the description of electronic properties $^{7}$. In addition, considerable work is in progress on the properties of the density matrix of $N$-particles systems 28,17 . The refinement of the $1-R D M$ relative to the pseudo experimental data have yielded many useful and very interesting results providing excellent information about chemical bonding, electrostatic potential, and orbital occupations $\frac{718 / 19}{\text {. }}$

To reconstruct the entire 1- $R D M$, several experiments need to be combined, because each one can only give partial information. To our best knowledge, no single experimental setup exists to directly determine the total density matrices ${ }^{7}$. X-ray and polarized neutron scattering make it possible to describe charge and spin densities in the position space, respectively, which represent the diagonal elements of the $1-R D M^{20} \underline{22}$. The magnetic and non-magnetic Compton scattering describe the electron densities in the momentum space, which are mostly related to the off-diagonal elements of the $1-R D M^{22 \mid 23}$. The off-diagonal elements of the 1-RDM bring information which complement the position space electron density giving by the diagonal elements. They directly provide the ground-state expectation value of the total electron kinetic energy per unit cell. The off-diagonal elements, which are connected to the electron cloud polarization and to the relative phases of the atomic orbitals participating in the most delocalized molecular wave functions. Up to now, several models have been proposed to refine only the diagonal elements of the 1 - $R D M$ using the

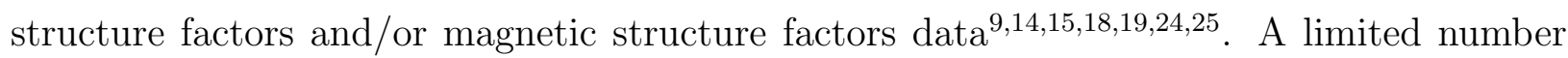
of works have made use of Compton scattering data; one to refine the wavefunction ${ }^{14}$ and others to reconstruct the momentum densities ${ }^{7113}$. In 2007 , one of us ${ }^{[15}$ proposed a strategy for refining a 1-RDM model from X-ray diffraction and deep inelastic scattering data. The model was adapted from the Hansen-Coppens pseudo-atomic description ${ }^{9}$ of electron density. A significant drawback of this approach was that no prior constraint was applied to the model to ensure that the $1-R D M$ is N-representable or even idempotent. In this paper, we will limit ourselves to the use of only two experimental techniques: polarized neutron scattering and magnetic Compton scattering. These two techniques provide information to reconstruct the entire spin resolved 1-RDM (1-SRDM). To our knowledge, a refinement 
of the 1-SRDM simultaneously using the data of magnetic structure factors (MSFs) and magnetic Compton profiles (MCPs) has not yet been studied. The aim of the present work is therefore to: (1) evaluate the quality of a combination of MCPs and MSFs data to reconstruct all the elements of the 1-SRDM, and (2) illustrate the changes made by MCPs not only to the off-diagonal elements of the $1-S R D M$ but also to its diagonal elements. The main stages of this model are: first, modelling a cluster using molecular ab-initio packages, and from the resulting ab initio data, the MSFs and the MCPs are computed. Secondly, the basis functions are rescaled by modifying the exponent coefficients for each atomic orbital. Finally, the spin population matrix is adjusted by varying the molecular orbitals occupation numbers. Optimization of the exponent coefficients and the occupation number parameters is carried out by minimizing the $\chi^{2}$ function (Eqs. 26 and 27) with respect to the pseudo experimental data using the Minuit code (developed at CERN) 26 . As a first test of our model, a simple molecular system, magnetic, with a small unit cell for rapid convergence, and with possibility to present an invariance of symmetry, need to be considered. A possible candidate is the urea molecule $\left(\mathrm{CO}\left(\mathrm{NH}_{2}\right)_{2}\right)^{27-29}$, which is planar (see Fig. 1), strongly anisotropic, and presents two mirror symmetries. The urea molecule is magnetic only by construction, the total spin is fixed to 1 . The molecular crystal of urea is an artificial system used to evaluate the performance of the process. Urea was chosen because it has been thoroughly investigated both theoretically and by different experimental techniques, among which X-ray diffraction and Compton scattering. In addition, the urea molecule contains different types of bonding, simple and double covalent bonds and, in its crystalline phase, hydrogen bonds. It is this diversity of bonds, together with the compactness of the molecule, which has guided our choice. However, the urea molecule is non-magnetic and, for our purpose it had to be modified to a total spin of 1. Additionally, since the influence of the crystalline environment effect needs to be unambiguously estimated, the unit cell was changed to bear a single molecule. The aim of the present work is to assess the versatility of the model and validate the refinement strategy, not to study the properties of a true urea crystal. The first results of our algorithm are presented focusing on the calculation of 1-SRDM, spin density, and MCPs. It is shown that the joint refinement model gives very satisfactory results to those obtained from periodic calculation of urea.

This paper is organized as follows: In Sec. II, we report the key equations for a density matrix and its relationship with various physical quantities. In Sec. III, we describe the 
generation of pseudo experimental data from periodic calculations of different molecular crystals. Then, in Sec. IV] we explain the different stages of the joint refinement model. Finally, in Sec. V, we present and discuss, the first results of our model and compare them to the periodic references. Conclusions are given in Sec. VI.

\section{GENERAL FRAMEWORK}

We have learned from quantum mechanics that a many-body system is completely characterized by its wavefunction. The $N$-electron wavefunction depends on $3 N$ variables. But, by increasing the system size, the $N$-electron wavefunction rapidly becomes too complicated to provide a simple physical picture of the system. Mostly (in particular for this work), the complete wavefunction is of no interest and the expectation values of physical observables are more significant. However, a $N$-particle system can be described by means of the $N$ electron density matrix, which bears a simpler and more direct physical meaning than the wavefunction itself. The $N$-electron density matrix is defined in terms of the $N$-electron wavefunction $\Psi\left(\mathbf{x}_{1}, \ldots, \mathbf{x}_{N}\right)$ :

$$
\Gamma^{N}\left(\mathbf{x}_{1}, \ldots, \mathbf{x}_{N} ; \mathbf{x}_{1}^{\prime}, \ldots, \mathbf{x}_{N}^{\prime}\right)=\Psi^{*}\left(\mathbf{x}_{1}^{\prime}, \ldots, \mathbf{x}_{N}^{\prime}\right) \Psi\left(\mathbf{x}_{1}, \ldots, \mathbf{x}_{N}\right),
$$

where $\mathbf{x}_{i}=\left(\mathbf{r}_{i}, s_{i}\right)$ represents the spin and position coordinates. In order to compute the expectation value of one-electron operator, we introduce the 1-RDM:

$$
\Gamma^{(1)}\left(\mathbf{x}_{1} ; \mathbf{x}_{1}^{\prime}\right)=N \int d \mathbf{x}_{2} \ldots d \mathbf{x}_{N} \Psi^{*}\left(\mathbf{x}_{1}^{\prime}, \mathbf{x}_{2}, \ldots, \mathbf{x}_{N}\right) \Psi\left(\mathbf{x}_{1}, \mathbf{x}_{2}, \ldots, \mathbf{x}_{N}\right) .
$$

In the position space, the diagonal elements of the 1-SRDM correspond to the spin density:

$$
\rho\left(\mathbf{r}_{1}\right)=\int\left[\delta\left(s_{1}-s^{\uparrow}\right)-\delta\left(s_{1}-s^{\downarrow}\right)\right] \Gamma^{(1)}\left(\mathbf{x}_{1} ; \mathbf{x}_{1}^{\prime}\right)_{\mathbf{x}_{1}=\mathbf{x}_{1}^{\prime}} d s_{1}
$$

The Fourier transform of the spin density gives the MSFs:

$$
F_{M}(\mathbf{Q})=\int\left[\delta\left(s_{1}-s^{\uparrow}\right)-\delta\left(s_{1}-s^{\downarrow}\right)\right] \Gamma^{(1)}\left(\mathbf{x}_{1} ; \mathbf{x}_{1}^{\prime}\right)_{\mathbf{x}_{1}=\mathbf{x}_{1}^{\prime}} e^{i \mathbf{Q} \cdot \mathbf{r}} d \mathbf{r},
$$

where $\mathbf{Q}$ is the scattering vector.

On the other hand, it is well-known that Compton scattering gives access to the electron density in the momentum space. In turn, the spin-resolved electron density in momentum space represents the off-diagonal elements of the 1-SRDM. The MCPs and the 1-SRDM are related, following this expression:

$$
J(\mathbf{u}, q)=\frac{1}{2 \pi \hbar} \times \int\left[\delta\left(s_{1}-s^{\uparrow}\right)-\delta\left(s_{1}-s^{\downarrow}\right)\right] \Gamma^{(1)}\left(\mathbf{x}_{1} ; \mathbf{x}_{1}^{\prime}\right)_{\mathbf{x}_{1}=\mathbf{x}_{1}^{\prime}} e^{i q \mathbf{u} \cdot \mathbf{r}^{\prime}} d \mathbf{r} d \mathbf{r}^{\prime}
$$


FIG. 1: Periodic array of urea with an intermolecular distance of $5 \AA$ (a) and $3 \AA$ (b). Their lattice parameters are, respectively, $\{\mathrm{a}=5 \AA, \mathrm{b}=10 \AA, \mathrm{c}=10 \AA$, $\left.\alpha=\beta=\gamma=90^{\circ}\right\}$ and $\left\{\mathrm{a}=3 \AA, \mathrm{b}=10 \AA, \mathrm{c}=10 \AA, \alpha=\beta=\gamma=90^{\circ}\right\}$.

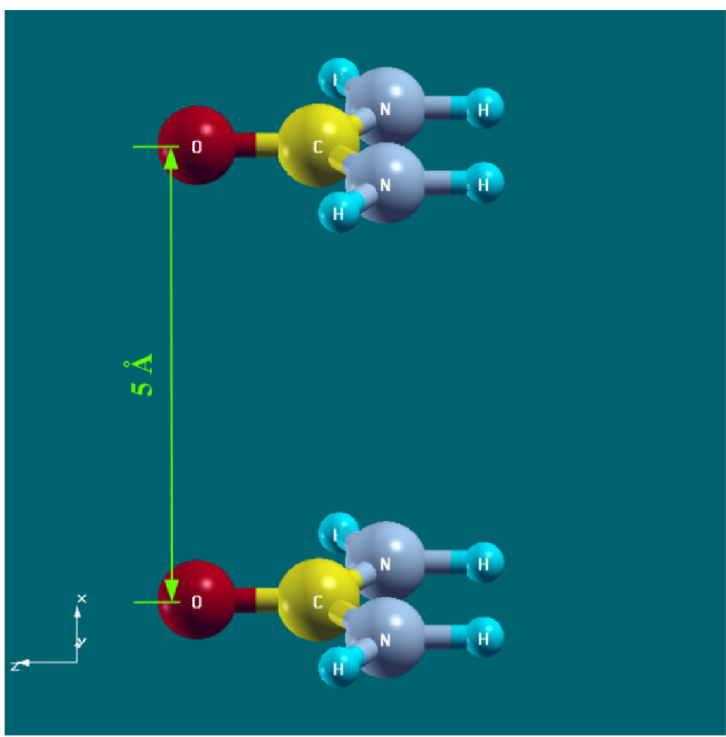

(a)

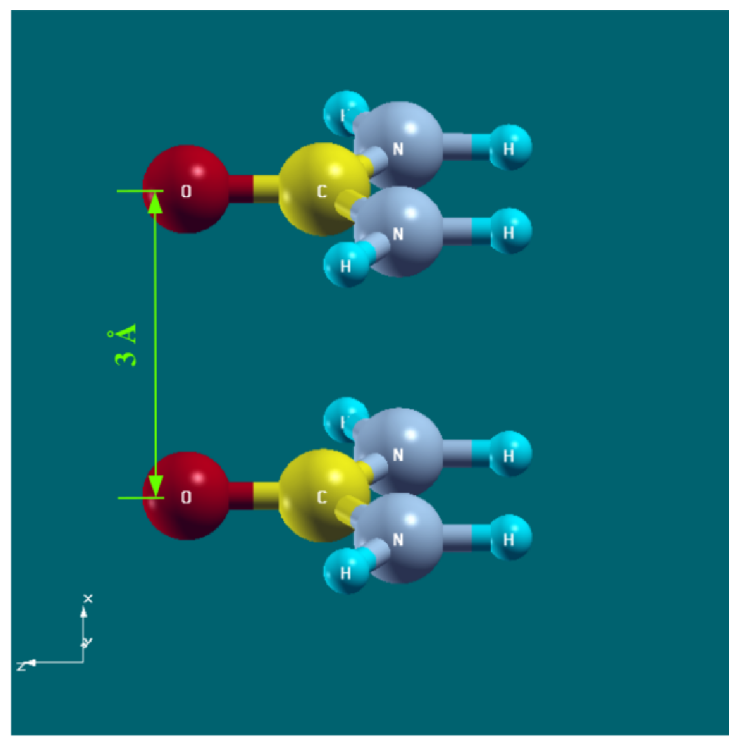

(b)

where $\mathbf{u}$ is a unit vector collinear to the inelastic scattering vector. The MCPs for momentum $q$ gives the spin density $n(\mathbf{p})$ in the momentum space projected onto the direction $\mathbf{u}$ :

$$
J(\mathbf{u}, q)=\int n(\mathbf{p}) \delta(q-\mathbf{p} \cdot \mathbf{u}) d \mathbf{p} .
$$

Therefore, to reconstruct the entire 1-SRDM, we need: (i) the MSFs, which describe the representation space, and (ii) the MCPs, which describe the momentum space. It's clear, that the two experimental techniques are highly complementary and give a complete physical picture of the spin density matrix.

\section{GENERATION OF PSEUDO EXPERIMENTAL DATA}

In order to test the performance of our model, we refined the 1 -SRDM relative to theoretical data obtained from periodic calculations. Once this procedure is validated, the model can safely be applied to genuine experimental data (and will be reported in a following paper). Because the calculation of the MSFs and the MCPs are standard features of its output, our periodic calculations are performed by the CRYSTAL14 packages $\sqrt{30} \sqrt[32]{3}$ using

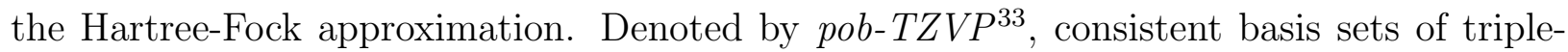


zeta valence $(T Z V)$ with polarization quality for main group elements and transition metals from row one to three have been used for periodic calculations. Two sets of calculations are considered for the periodic array of urea: the first set neglects the interaction between the molecules, separated by $5 \AA$ along the X-axis direction (see Fig. 11a). In the second, the interaction between molecules are included by reducing to $3 \AA$ the inter-molecular distance along the $\mathrm{X}$-axis direction (see Fig. 1.b). Note that the intermolecular interactions in the artificial crystal of urea are quite different from the true one. In the actual urea crystal, molecules interact in a perpendicular conformation, while in the artificial one they interact in a parallel conformation. The urea molecule is composed of 8 atoms; the central carbon atom makes a strong covalent bond with the oxygen atom. It has two symmetrical groups of $\mathrm{NH}_{2}$ around the central axis $\mathrm{O}=\mathrm{C}$. As a reminder, in this work, the urea molecule is magnetic only by construction; the total spin on the molecule is considered in its triplet state.

In the present work, the refinement was performed with respect to 12 directions of MCPs and 500 MSFs generated from these periodic calculations. To be consistent with usual experimental data, a statistical noise generated from normal Gaussian distribution is added to the computed periodic data. The amplitude of this noise is determined from that considered in usual experimental measurements. For the MSFs data, the error bar of each point corresponds to $10 \%$ of its corresponding MSF amplitude. For the MCPs data, the error bar of each point corresponds to the square root of its corresponding MCP amplitude.

\section{A JOINT REFINEMENT MODEL}

The different stages of the joint refinement model are explained in this section, and, in particular, an illustration of the self-consistent procedure of the code will be reported. Our model combines both the MSFs and the MCPs to refine the 1-SRDM. The joint refinement model is based on four main stages: (1) modelling a cluster using molecular ab-initio packages, (2) computation of the MSFs and the MCPs, (3) variation of the basis functions, and (4) variation of occupation numbers. 


\section{A. Ab initio calculation: Modeling a molecule/cluster}

The molecular calculation conducted in this work is performed using the GAUSSIAN 09 packages $^{34}$ by means of the Hartree-Fock approximation. The main objective of this model is to reconstruct the spin density matrices of a periodic array using the corresponding experimental data from a simple molecular or cluster model and with a limited basis set. Therefore, $3-21+G^{*}$ was used, i.e. $3-21 G$ basis set ${ }^{35-40}$ with polarization functions ${ }^{41}(d)$ on second row atoms only, supplemented by diffuse functions ${ }^{42}$. Very different basis sets are considered for both molecular and periodic calculations in order to emphasize how the initial molecular basis set (as minimal as possible) can be improved and can adapt to a particular crystalline configuration using the pseudo-experimental information (derived from the large basis set computation). Obviously, once the model and the refinement strategy are validated, we intend to apply this method to genuine experimental data and it will be legitimate (and desirable) to use much better and flexible basis sets for the model. In the following, we present the output of the GAUSSIAN $\operatorname{code}^{34}$ that serves as an input of our algorithm. In this context, the molecular orbitals are constructed by a linear combination of the basis functions as

$$
\Phi_{i}(\mathbf{r})=\sum_{j=1} C_{i, j} \chi_{j}(\mathbf{r})
$$

where $C_{i, j}$ are the molecular orbital coefficients, $\chi_{j}(\mathbf{r})$ are functions in the minimal basis sets. In the GAUSSIAN framework, the basis functions are defined as a linear combination of gaussian functions

$$
\chi_{j}(\mathbf{r})=\sum_{k=1} d_{j, k} N\left(\alpha_{k}\right)\left(x-A_{x_{k}}\right)^{a_{k}}\left(y-A_{y_{k}}\right)^{b_{k}}\left(z-A_{z_{k}}\right)^{c_{k}} e^{-\alpha_{k}\left(\mathbf{r}-\mathbf{R}_{k}\right)^{2}}
$$

where $a_{k}, b_{k}$ and $c_{k}$ control the angular moments $L$, where $L=a_{k}+b_{k}+c_{k}, d_{j, k}$ are the contraction coefficients of the $j$ th orbital and their corresponding $k$ th gaussian function. Once the expansion of the molecular orbitals is obtained, the 1-SRDM can be expressed as a functional of natural molecular orbitals $\Phi_{i}(\mathbf{r})$ and their respective occupation numbers $n_{i}$, which represent the eigenfunctions and eigenvalues, respectively, as

$$
\Gamma^{(1)}\left(\mathbf{r}, \mathbf{r}^{\prime}\right)=\sum_{i} n_{i} \Phi_{i}(\mathbf{r}) \Phi_{i}\left(\mathbf{r}^{\prime}\right)
$$


Using Eqs. 7 and 9, the 1-SRDM can be expressed as

$$
\Gamma^{(1)}\left(\mathbf{r}, \mathbf{r}^{\prime}\right)=\Gamma_{\uparrow}^{(1)}\left(\mathbf{r}, \mathbf{r}^{\prime}\right)-\Gamma_{\downarrow}^{(1)}\left(\mathbf{r}, \mathbf{r}^{\prime}\right)=\sum_{j, k} P_{j, k} \chi_{j}(\mathbf{r}) \chi_{k}\left(\mathbf{r}^{\prime}\right),
$$

where the spin population matrix is defined as a function of the occupation numbers and the molecular coefficients for each spin state:

$$
P_{j, k}=\sum_{i=1} n_{i}^{\uparrow} C_{i, j}^{\uparrow} C_{i, k}^{\uparrow}-\sum_{i=1} n_{i}^{\downarrow} C_{i, j}^{\downarrow} C_{i, k}^{\downarrow} .
$$

The $1-S R D M$ is thus expressed in terms of the spin population matrix $P_{j, k}$ and the product of two basis functions. The variation of these quantities will be discussed in the next sections.

\section{B. Computation of the magnetic structure factors and the magnetic Compton} profiles

Once the $1-S R D M$ of the system is known, all one-electron observables of the system can be expressed as a function of the spin population matrix and the product of two basis functions. We start by considering the MSFs $F_{M}(\mathbf{Q})$, which are defined as Fourier transforms of the diagonal elements of the 1-SRDM. Using Eqs. 4 and 10, the expansion of the MSFs is written as:

$$
F_{M}(\mathbf{Q})=\sum_{j, k} P_{j, k} \int \chi_{j}(\mathbf{r}) \chi_{k}(\mathbf{r}) e^{i \mathbf{Q} \cdot \mathbf{r}} d \mathbf{r}
$$

The MSFs are defined as a sum of the product of the spin population matrix and the Fourier transform of the product of two basis functions.

By inserting Eq. 10 into Eqs. 5 and 6, the MCPs can be expressed as follows

$$
J(\mathbf{u}, q)=\frac{1}{2 \pi} \sum_{j, k} P_{j, k} \iint\left[\int S_{j, k}(\mathbf{r}) e^{-i \mathbf{p} \cdot \mathbf{r}} d \mathbf{r}\right] e^{i \mathbf{p} \cdot \mathbf{u} t} d \mathbf{p} e^{-i q t} d t,
$$

where

$$
S_{j, k}(\mathbf{r})=\int \chi_{j}\left(\mathbf{r}^{\prime}\right) \chi_{k}\left(\mathbf{r}^{\prime}+\mathbf{r}\right) d \mathbf{r}^{\prime}
$$

The MSFs as well as the MCPs are functionals of 1-SRDM and therefore are a functional of the spin population matrix and the product of two basis functions.

\section{Variation of the basis functions}

In order to obtain accurate $1-S R D M$, we first modified the radial extension of each atomic orbital. This makes it possible to take into account the diffuse effect. To do this, the 
basis functions are rescaled by varying the exponent coefficients of each atomic orbital $j$

$$
\begin{aligned}
\left(\{\alpha\} \Longrightarrow\left\{\alpha_{0}\right\} \times \zeta_{j}\right) \text { as } & \\
\chi_{j}\left(\zeta_{j}, \mathbf{r}\right)= & \sum_{k=1} d_{j, k} N\left(\zeta_{j} \alpha_{0, k}\right) \times \\
& \left(x-A_{x_{k}}\right)^{a_{k}}\left(y-A_{y_{k}}\right)^{b_{k}}\left(z-A_{z_{k}}\right)^{c_{k}} e^{-\zeta_{k} \alpha_{0, k}\left(\mathbf{r}-\mathbf{R}_{k}\right)^{2}} .
\end{aligned}
$$

In such a minimization, the variation of atomic orbitals is performed under the following conditions:

$$
\left\{\begin{array}{l}
0.8 \lesssim \zeta_{j} \lesssim 1.2 \\
\int d \mathbf{r} \Phi_{j}(\mathbf{r}) \Phi_{k}(\mathbf{r})=\delta_{j, k}
\end{array}\right.
$$

The first condition controls the extension of the atomic orbitals. The second condition provides a molecular orbital orthonormal set, generated by the ab-initio packages after each variation of the $\zeta$ coefficients. The variation of the basis functions can clearly be observed in the expansions of the MSFs

$$
F_{M}(\mathbf{Q})=\sum_{j, k} P_{j, k} \int \chi_{j}\left(\zeta_{j}, \mathbf{r}\right) \chi_{k}\left(\zeta_{k}, \mathbf{r}\right) e^{i \mathbf{Q} \cdot \mathbf{r}} d \mathbf{r}
$$

and the MCPs

$$
J(\mathbf{u}, q)=\frac{1}{2 \pi} \sum_{j, k} P_{j, k} \iint\left[\int S_{j, k}(\{\zeta\}, \mathbf{r}) e^{-i \mathbf{p} \cdot \mathbf{r}} d \mathbf{r}\right] e^{i \mathbf{p} \cdot \mathbf{u} t} d \mathbf{p} e^{-i q t} d t,
$$

where

$$
S_{j, k}(\{\zeta\}, \mathbf{r})=\int \chi_{j}\left(\zeta_{j}, \mathbf{r}^{\prime}\right) \chi_{j}\left(\zeta_{k}, \mathbf{r}^{\prime}+\mathbf{r}\right) d \mathbf{r}^{\prime}
$$

\section{Variation of the spin population matrix}

After finding the best basis functions, a variation of the spin population matrix is performed by varying the occupation numbers for a selection of states and for each spin configuration:

$$
n_{i}^{\sigma}=n_{i, 0}^{\sigma}+\delta n_{i}^{\sigma}
$$

where $n_{i, 0}^{\sigma}$ is the initial occupation number of the $i$ th state, and $\sigma=[\uparrow, \downarrow]$ represents their spin state. Changes in the occupation number concerns only a few states in the vicinity of the Fermi level. The variations of the occupation numbers are performed under the N-representability conditions for $1-S R D M$ :

$$
\left\{\begin{array}{l}
0 \leq n_{i}^{\sigma} \leq 1 \\
\sum_{i=1}\left(n_{i}^{\uparrow}+n_{i}^{\downarrow}\right)=N_{\text {electron }}, \\
\sum_{i=1}\left(n_{i}^{\uparrow}-n_{i}^{\downarrow}\right)=N_{\text {spin }} .
\end{array}\right.
$$


The first reflects the Pauli principle, while the second and the third conditions represent electron number conservation and spin number conservation, respectively. The second condition is enforced by minimizing the following quantity

$$
C\left(\chi^{2}(\{n\})\right)=\sum_{j} \log \left(\chi_{j}^{2}(\{n\})\right)-\mu_{1}\left(\sum_{i=1} n_{i}^{\uparrow}-N_{\text {electron }}^{\uparrow}\right)-\mu_{2}\left(\sum_{i=1} n_{i}^{\downarrow}-N_{\text {electron }}^{\downarrow}\right),
$$

where $j$ stands for a given experiment (polarized neutron scattering, magnetic Compton scattering), and $\mu_{1}, \mu_{2}$ are Lagrange multipliers. The first term of Eq. 22 performs the pseudo experimental data and the resulting data from the model (explained in the next part). However, the second and the third part of the Eq. 22 ensure the electron number

conservation for each spin state. A dramatic consequence of the last two parts of Eq. 22 is that it satisfies the third condition of Eq. 21: it imposes the spin number conservation. Changes in the occupation numbers lead to a variation of the spin population matrix as follows:

$$
P_{j, k}=P_{j, k}^{0}+\delta P_{j, k}
$$

with

$$
\left\{\begin{array}{l}
P_{j, k}^{0}=\sum_{i=1} n_{i, 0}^{\uparrow} C_{i, j}^{\uparrow} C_{i, k}^{\uparrow}-\sum_{i=1} n_{i, 0}^{\downarrow} C_{i, j}^{\downarrow} C_{i, k}^{\downarrow}, \\
\delta P_{j, k}=\sum_{i=1} \delta n_{i}^{\uparrow} C_{i, j}^{\uparrow} C_{i, k}^{\uparrow}-\sum_{i=1} \delta n_{i}^{\downarrow} C_{i, j}^{\downarrow} C_{i, k}^{\downarrow} .
\end{array}\right.
$$

The first term represents the initial spin population matrix obtained from the ab initio calculation for the finite system, using the GAUSSIAN code ${ }^{34}$, expressed in terms of the initial occupation numbers and the molecular coefficients for each spin state. The second term represents the variation of the spin population matrix defined as a function of the variation of the occupation numbers and the molecular coefficients for both spin states.

\section{E. Illustration of SCF procedure of the joint refinement code}

The model depends on a set of parameters $\{x\}$. In other words, we wish to find the set $\{x\}$ that minimizes the following quantity $C\left(\chi^{2}\right)=\sum_{Y} \log \left(\chi_{Y}^{2}\right)$. The so-called $\chi^{2}$ is defined as follows:

$$
\chi_{Y}^{2}(\{x\})=\sum_{i} \frac{\left|Y_{i}^{m}(\{x\})-Y_{i}^{c}(\{x\})\right|^{2}}{\sigma_{i}^{2}},
$$

where $i$ runs over all the measured/calculated MSFs $\left(Y^{m} / Y^{c}=F^{m} / F^{c}\right)$ or $\operatorname{MCPs}\left(Y^{m} / Y^{c}=\right.$ $\left.J^{m} / J^{c}\right)$ with $\sigma_{i}$, the estimated standard deviation associated with $Y_{i}^{m}$. The minimization 
FIG. 2: Illustration of SCF procedure of our code.

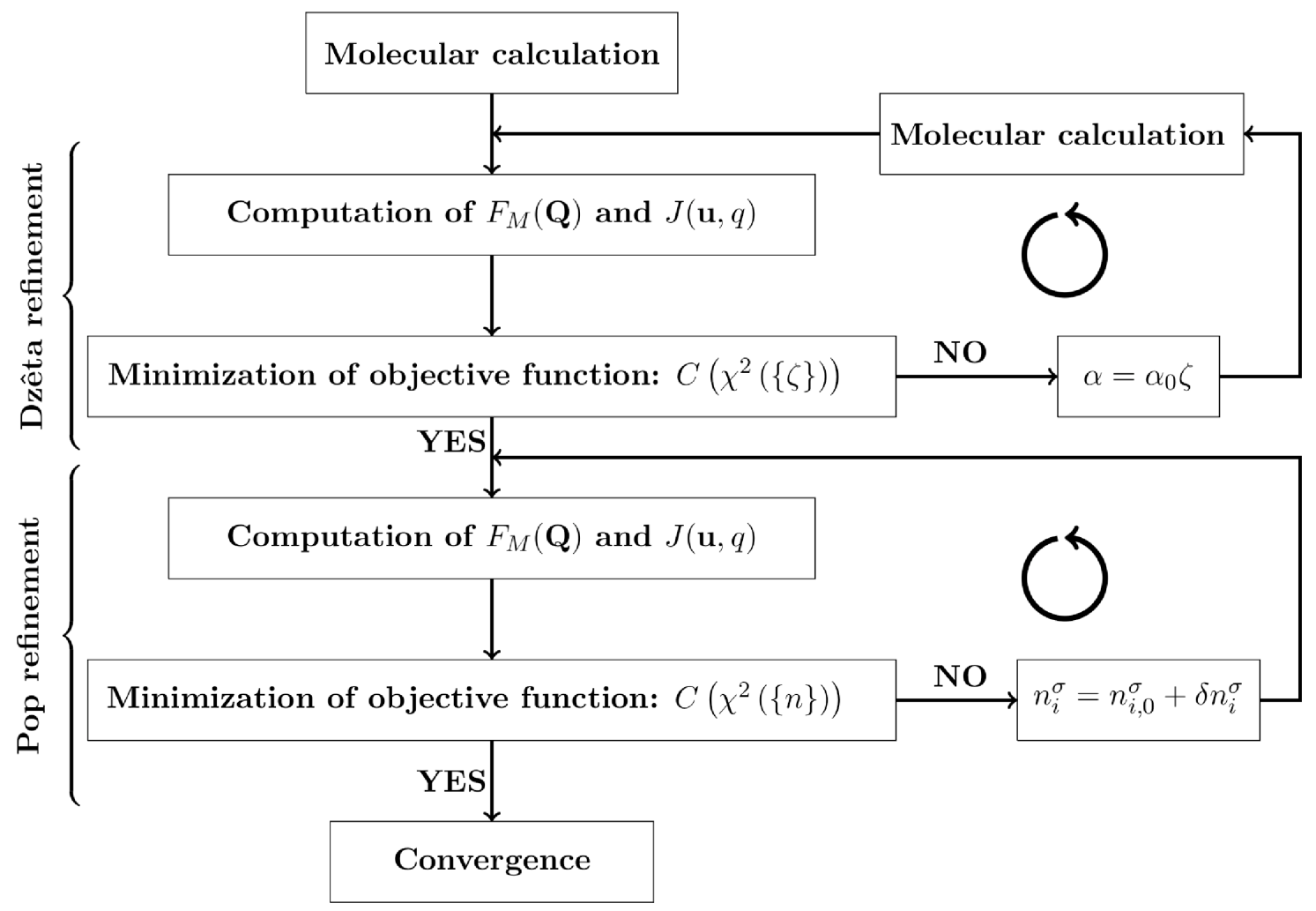

of function $C$ with respect to the parameters $\{x\}$ was performed by the MINUIT code ${ }^{26}$. In our algorithm, this minimization is performed twice separately, first for the basis function rescaling optimization via $\{\zeta\}$ and then for the variation of the spin population matrix via $\{n\}$.

For the variation of the basis function (Dzêta refinement), the minimizing function $C$ is defined as follows:

$$
C\left(\chi^{2}(\{\zeta\})\right)=\log \left(\chi_{J}^{2}(\{\zeta\})\right)+\log \left(\chi_{F}^{2}(\{\zeta\})\right)
$$

where $F$ and $J$ are, respectively, the MSFs and the MCPs. However, the expression of the $C$ function to vary the spin population matrix (Pop refinement) is given by:

$$
C\left(\chi^{2}(\{n\})\right)=\log \left(\chi_{J}^{2}(\{n\})\right)+\log \left(\chi_{F}^{2}(\{n\})\right) .
$$

At this point, it is useful to briefly recapitulate the different steps of our joint refinement model (see Fig. 2). It consists of the following steps:

1. Perform an ab-initio calculation using local atomic orbitals as a basis set. An atomic orbital is defined as a linear combination of gaussian functions. Here, we use the 
GAUSSIAN 09 packages ${ }^{34}$, but other codes like the GAMESS code ${ }^{43}$ could also be used.

2. Compute the MSFs and the MCPs.

3. Rescale the basis functions by varying the exponent coefficients. In other words, we minimize the $C$ function Eq. 26 with respect to the parameters $\{\zeta\}$ (exponent coefficients). The variation of the exponent coefficients is followed by a molecular calculation. Then the new MSFs and the MCPs are computed and this procedure is continued until convergence.

4. Find the most probable values for the parameters $\{n\}$ that minimize the $C$ function Eq. 27. After each variation of the occupation numbers, we calculate the new MSFs and the MCPs and we continue the procedure in a self-consistent way until convergence.

Results obtained with the above algorithm are now discussed in sec. $\mathrm{V}$.

\section{RESULTS AND DISCUSSIONS}

In this work, to check the robustness of the joint refinement model, we have studied two possibilities of a urea molecular crystal. We first consider a periodic array of isolated molecules, where the corresponding lattice parameters are set to: $a=5 \AA, b=10 \AA$ and $c=10 \AA$. Then, to evaluate how such a simple model can account for the changes in the properties induced by intermolecular interactions, the $a$ value is changed to $3 \AA$. Remember that the parallel intermolecular interactions in this case are quite different from the true one. For both configurations, the total spin on each molecule is fixed to 1 . In the present study we have chosen to also show the results obtained from a refinement on MSFs only, to illustrate in contrast the potential benefits brought by a joint refinement. In addition, we present intermediate results obtained from Dzêta refinements. This will highlight the effect provided by each set of parameters. The final results being presented are obtained from a refinement of the spin population matrix following the Dzêta refinement, called Dzêta + Pop refinement. 


\section{A. Periodic array of isolated urea molecules}

In this section, we consider an isolated molecule of urea for both molecular and periodic calculations. The periodic calculations are performed at the Hartree-Fock approximation level using one of the best basis sets available in CRYSTAL 14 packages $^{30}+32$, the pop-TZVP basis sets 33 . We aim to reproduce the properties of crystalline urea, using a molecular calculation within a simple basis set $\left(3-21+G^{*}\right)^{35} \underline{42}$ as a starting point of our model. The calculated spin densities in the plane of the urea molecule are displayed in Fig. 3 for comparison with the results of the refinement model on periodic pseudo data. By comparing the molecular and the periodic spin density maps, the differences are mostly seen on the oxygen atom. For the periodic calculation, the corresponding spin density exhibits a negative spin distribution around the oxygen nucleus in the form of two lobes directed along the $\mathrm{O}=\mathrm{C}$ direction. However, the molecular calculation shows in addition a small positive spin population between the two lobes. The negative lobes are slightly larger in the molecular calculation than in the periodic one. Moreover, the negative spin distribution (red contours) along the $\mathrm{C}-\mathrm{N}$ bonds are also different. Thus results from the polarization functions. These mismatch are due to the difference in the basis functions used in both calculations. The molecular and the periodic spin distributions of the remaining atoms of the molecule compare extremely well.

Our results show that the spin densities obtained from Dzêta refinement on MSFs only are slightly affected: the positive spin distribution in the vicinity of the oxygen nucleus disappears. This is in agreement with the crystal results. However, the negative contours between the nitrogen and the carbon atoms are unchanged compared to the molecular calculation and it remains at variance with the periodic results. By combining the MCPs with the MSFs, the negative distribution along the $\mathrm{C}-\mathrm{N}$ bonds and the negative distribution around the oxygen atom obtained from Dzêta refinement are consistent with the corresponding spin distribution obtained from the periodic calculation. However, the resulting spin densities obtained from both Dzêta + Pop refinement relative to MSFs only and MSFs + MCPs are at variance with the periodic spin density. This is because the basis sets are different. Therefore, only the variation of the basis functions are required in this case. Fig. 4 shows the computed 1-SRDM maps from molecular (a) and periodic (b) calculations (upper panel) for comparison with the results of the refinement model on periodic pseudo data: the middle 
FIG. 3: V A Spin density maps in the plane of the urea molecule (YZ). Upper panel: Computed spin resolved electron densities for molecular (a) and periodic calculations (b). Second panel: Maps of the Dzêta $(\mathbf{c})$ and Dzêta + Pop (d) refined spin density with respect to the MSFs only. Lower panel: The same as the second panel but obtained by means of the joint refinement strategy (MSFs + MCPs). Contours at intervals of $\pm 0.01 \times 2^{n} \mu_{B} \AA^{-3}(\mathrm{n}=0-12)$ : positive and negative contours are, respectively, blue solid lines and red dashed lines, and neutral contours are green dashed lines.

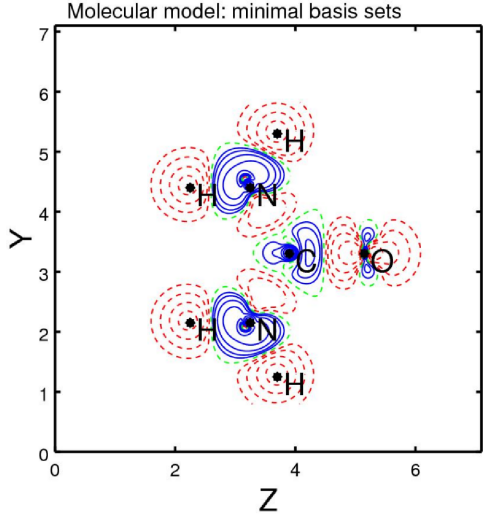

(a)

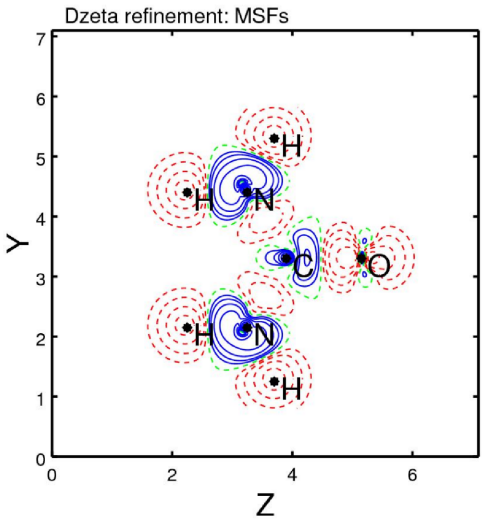

(c)

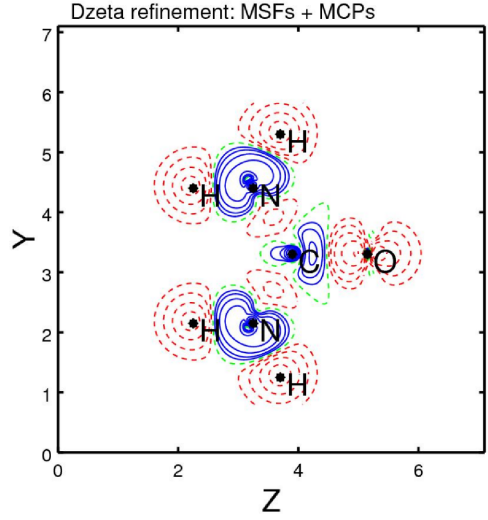

(e)

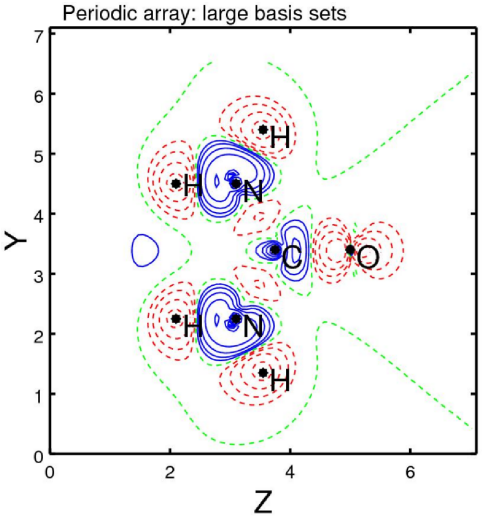

(b)

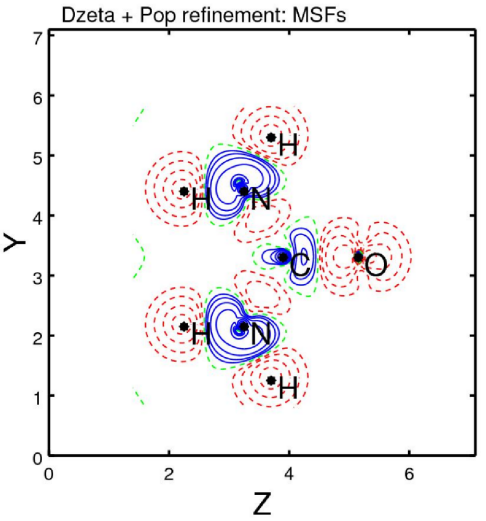

(d)

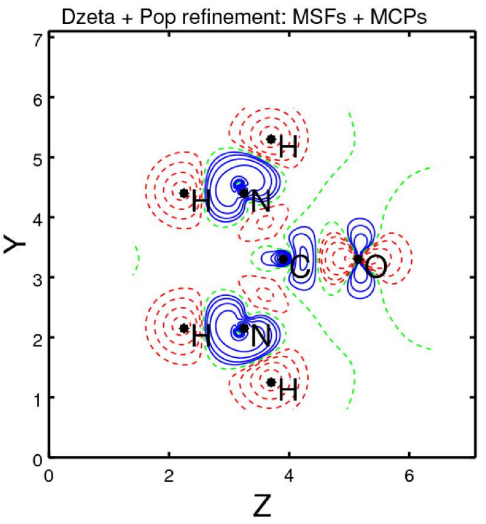

(f) 
FIG. 4: VA 1-SRDM $\Gamma^{(1)}\left(\mathbf{r}, \mathbf{r}^{\prime}\right)$ of the urea molecule is plotted along the $\mathrm{O}=\mathrm{C}-\mathrm{N}-\mathrm{H}$ path (in the plan of the molecule). The upper panel shows the molecular (a) and periodic (b) computation of the 1-SRDM. The second panel shows the 1-SRDM after a Dzêta (c) and Dzêta + Pop $(\mathbf{d})$ refinement relative to the MSFs only. The lower panel is the same as the second panel, but by means the joint refinement (MSFs and MCPs). Contours at intervals of $\pm 0.01 \times 2^{n} \mu_{B} \AA^{-3}(\mathrm{n}=0-20)$ : positive and negative contours are, respectively, blue solid lines and red dashed lines, and neutral contours are green dashed lines.

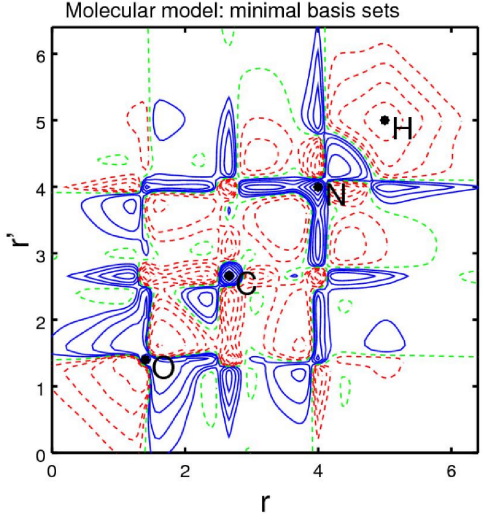

(a)

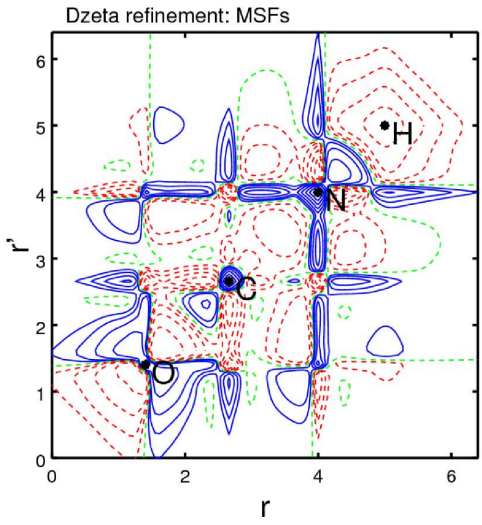

(c)

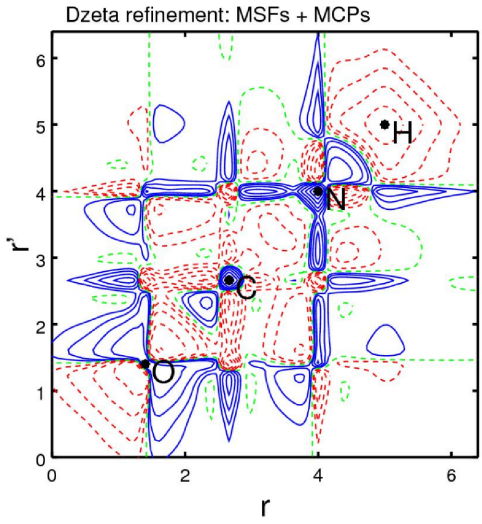

(e)

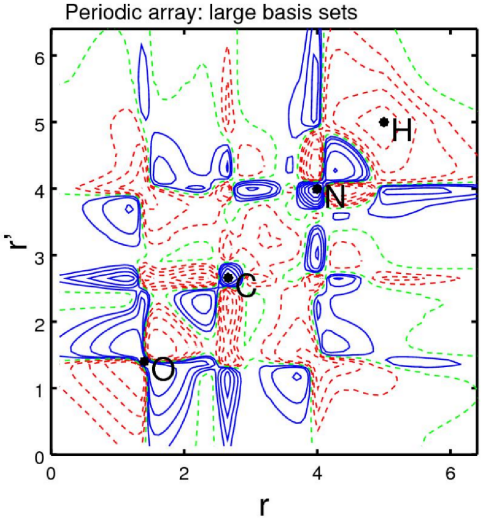

(b)

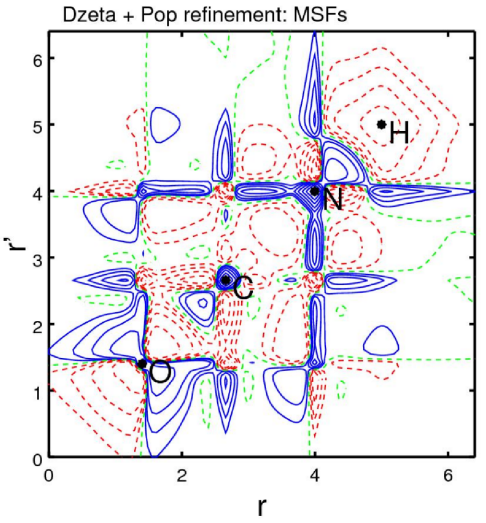

(d)

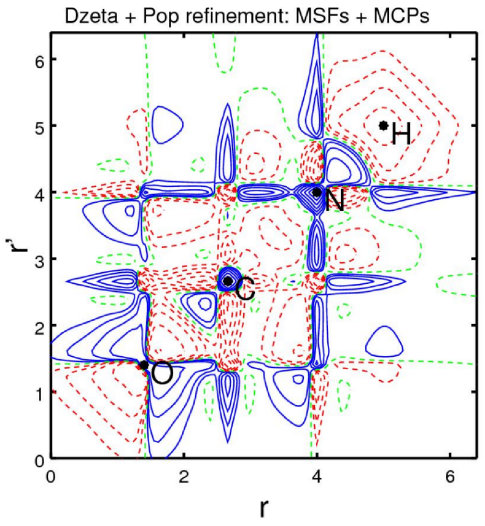

(f) 
panel shows the resulting 1-SRDM from refinement on MSFs only, while the lower panel shows the resulting 1-SRDM from joint refinement model. The 1-SRDM maps $\Gamma^{(1)}\left(\mathbf{r}, \mathbf{r}^{\prime}\right)$ are plotted as a function of $\mathbf{r}$ and $\mathbf{r}$ ', where both follow the $\mathrm{O}=\mathrm{C}-\mathrm{N}-\mathrm{H}$ path. By comparing the molecular and the periodic calculations of density matrices (see upper panel of Fig. 4), the differences appear on the diagonal parts (as discussed above on the spin densities) and on the off-diagonal parts, mainly in the vicinity of $\Gamma^{(1)}\left(\mathbf{r}=1.6, \mathbf{r}^{\prime}=3.9\right), \Gamma^{(1)}\left(\mathbf{r}=2.8, \mathbf{r}^{\prime}=3.9\right)$ points. The resulting 1-SRDM from a joint refinement model confirms that the MCPs information improves the diagonal elements corrected by the MSFs. The spin distributions on the diagonal parts compare very well with the periodic calculation. A weak influence of the MCPs is observed on the off-diagonal elements of the 1-SRDM, mainly around the $\Gamma^{(1)}\left(\mathbf{r}=3.9, \mathbf{r}^{\prime}=2.8\right)$ point. The comparison of the 1-SRDM maps obtained from Dzêta and Dzêta + Pop refinements relative to MSFs and MSFs + MCPs confirms that the corrections made to the 1-SRDM are due to the variation of the basis functions. In this section, although the effect of MCPs information in this system is small, we have shown that the joint refinement gives more accurate results than the refinement on MSFs only. We confirmed that the MSFs improve the diagonal elements of the 1-SRDM. Moreover, we have shown that the MCPs affect the diagonal and off-diagonal elements of the 1-SRDM. The MSFs and the MCPs are highly complementary and give very satisfactory results in agreement with the periodic pseudo data. Because the urea molecule is isolated in both calculations, the MCPs are slightly different, as can be seen in Fig. 5. In this work, 12 profiles were considered in the three planes XY, XZ and YZ. MCPs, for [ $\left[\begin{array}{lll}1 & 0 & 0\end{array}\right],\left[\begin{array}{lll}1 & 1 & 0\end{array}\right]$ and $\left[\begin{array}{lll}0 & 1 & 0\end{array}\right]$ directions, obtained from molecular calculations, refinements or periodic computation as reported in Fig. 5. Our results show that the molecular and the periodic calculations give in general very

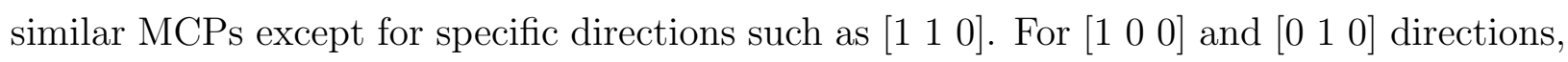
the resulting MCPs from joint refinement are slightly changed from those of the molecular calculation and, of course, in agreement with the periodic MCPs. For the $\left[\begin{array}{lll}1 & 1 & 0\end{array}\right]$ direction, the difference between the molecular and the periodic calculation is slightly reduced, mainly due to the variation of the basis functions. The remaining discrepancy can be attributed to the limitation of degrees of freedom in this case: the molecular model is very similar to the periodic system, it is mainly a difference of basis sets. 
FIG. 5: VA MCPs computed on the following directions $\left[\begin{array}{lll}1 & 0 & 0\end{array}\right],\left[\begin{array}{lll}1 & 1 & 0\end{array}\right]$ and $\left[\begin{array}{lll}0 & 1 & 0\end{array}\right]$ as a function of the momentum q. For each direction, the resulting MCPs from periodic calculation (CRYS 14, red solid lines) is compared to resulting MCPs from, molecular calculation (G09, black solid lines), Dzêta refinement (Dzeta Ref, green solid lines) and Dzêta + Pop refinement (Dzeta+Pop Ref, blue dashed lines).
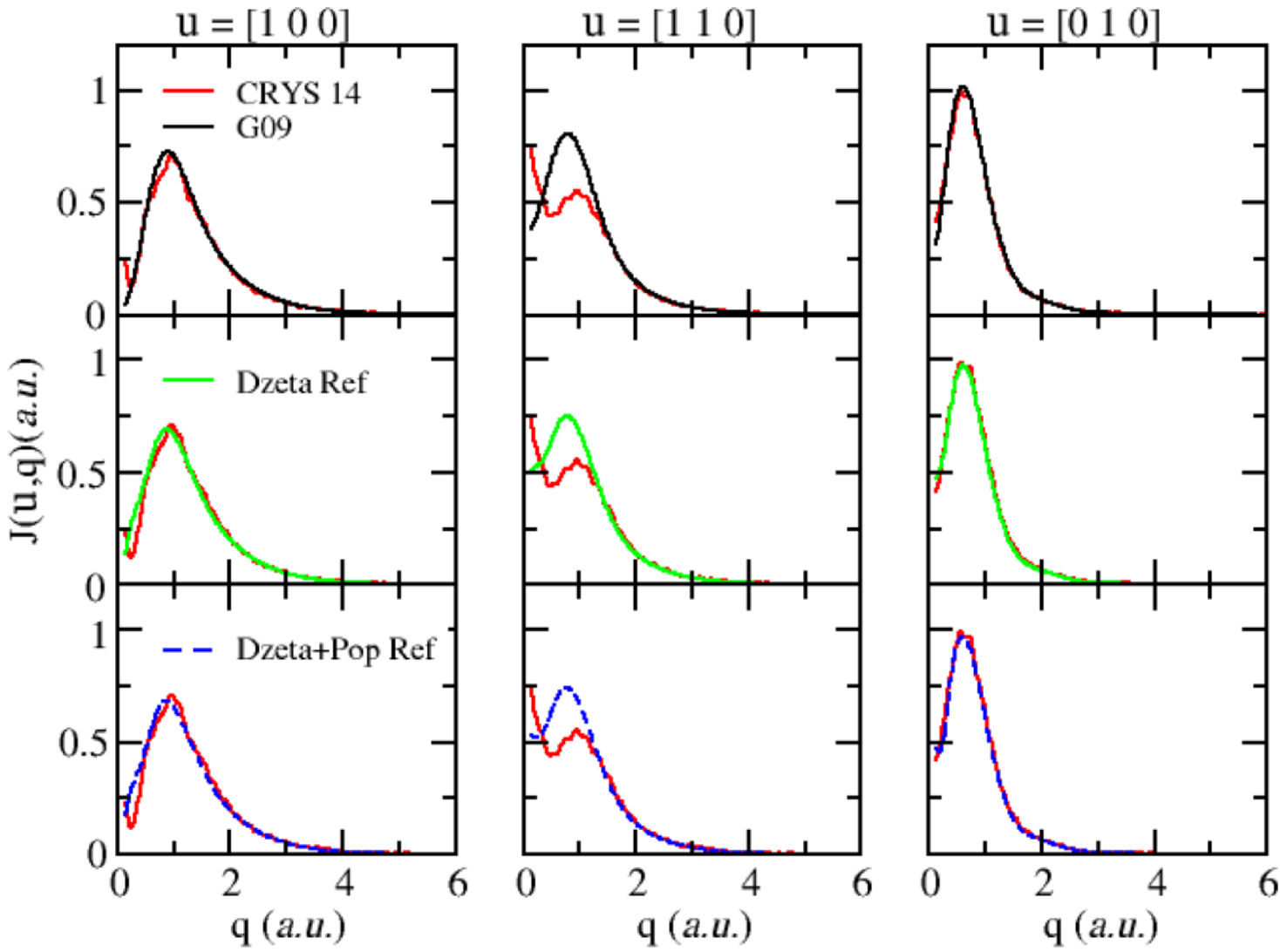

\section{B. Periodic array of interacting urea molecules}

To show the potential benefits brought by the MCPs, an array with interacting urea molecules is now considered (see Fig. 1, right panel). The interactions between molecules do not exist in the molecular calculation. Therefore, the computed MCPs obtained from molecular and periodic calculations are necessarily different, as shown later by means of the Compton profiles analysis. The difference is also expected to show up on the spin density performed from molecular and periodic computations. Fig. 6 reports the computed spin densities in the plane of the urea molecule compared with the resulting spin densities from the refinement model on periodic pseudo data. By comparing the molecular and 
FIG. 6: VB Spin density maps in the plane of the urea molecule (YZ). Upper panel: Computed spin resolved electron densities for molecular (a) and periodic calculations (b). Second panel: Maps of the Dzêta (c) and Dzêta + Pop (d) refined spin density with respect to the MSFs only. Lower panel: The same as the second panel but obtained by means of the joint refinement strategy (MSFs + MCPs). Contours at intervals of $\pm 0.01 \times 2^{n} \mu_{B} \AA^{-3}(\mathrm{n}=0-12):$ positive and negative contours are, respectively, blue solid lines and red dashed lines, and neutral contours are green dashed lines.

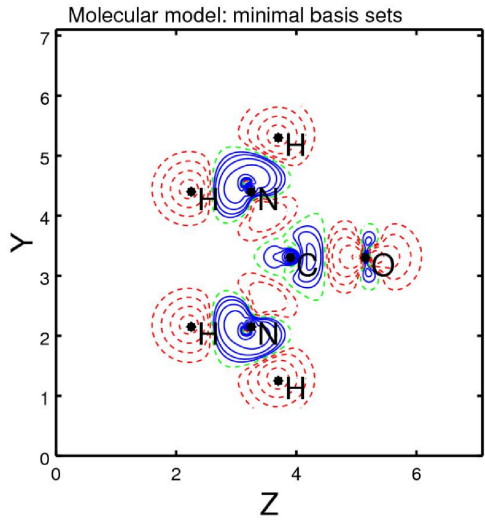

(a)

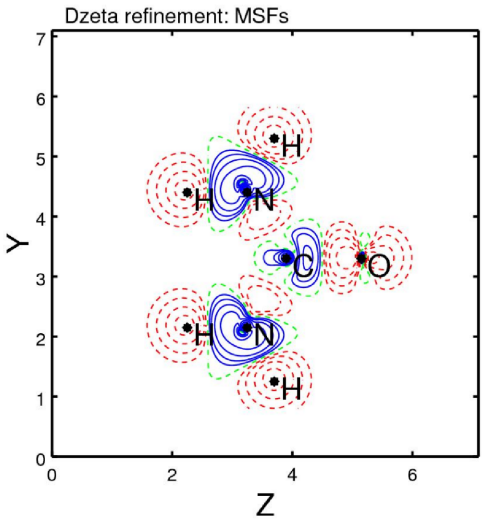

(c)

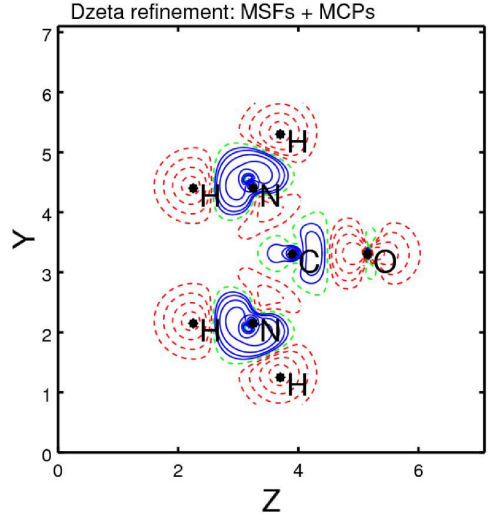

(e)

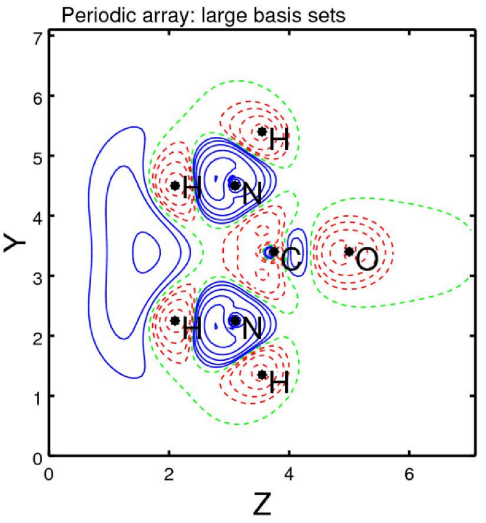

(b)

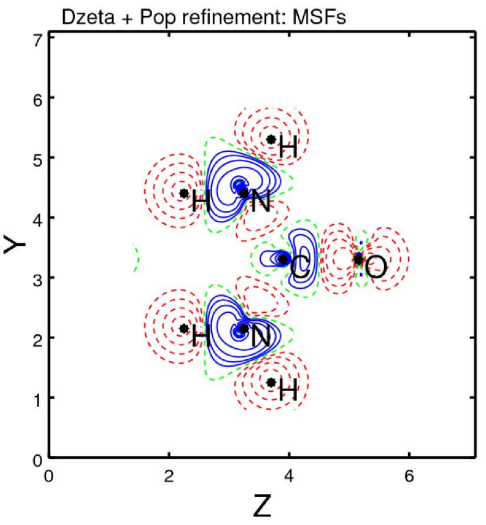

(d)

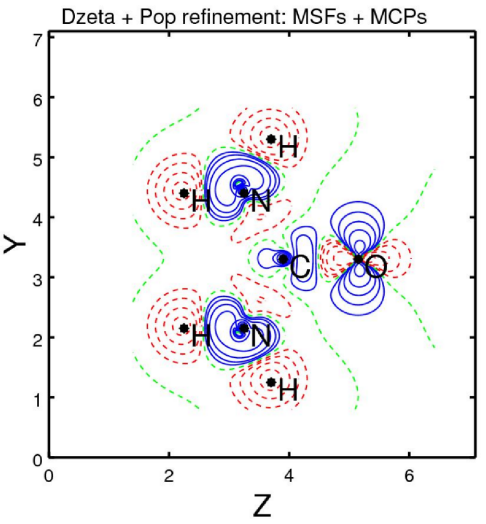

(f) 
the periodic spin density maps (see upper panel of Fig. 6), differences are clearly visible around the oxygen and the carbon atoms. In addition, positive contours appear close to the hydrogen atoms due to the effect of the second molecule. For the periodic calculation, the negative spin distribution on the oxygen atom is spherical. For the molecular calculation, the corresponding spin density shows two negative lobes with small positive contours in the vicinity of the nucleus. The spin density around the oxygen and carbon atoms are strongly affected upon interaction with neighboring molecules. Our results show that the MSFs slightly affect the spin density in the plane of the molecule; the positive contours around the oxygen nucleus have disappeared and the negative contours along the $\mathrm{C}-\mathrm{N}$ bonds are barely changed. The refined spin densities relative to the MSFs only are at variance with the periodic results. It's also clear that the resulting spin densities from joint refinement do not reproduce with the periodic results, but small corrections made by the model are observed, such as the broadening of negative contours located along the C-N bonds. In addition, the positive contours on the carbon atom are consistent with those obtained by the periodic calculation. The strong effect of the nearest molecules on the carbon and the oxygen atoms prevents our model reproducing the spin distribution of these atoms with good accuracy. To show the efficiency of this model, we studied the $\pi$ electron distribution by computing the spin electron distribution in the plane perpendicular to the molecule along the X-axis direction through each bond. Fig. 7 shows the spin density maps through the $\mathrm{O}=\mathrm{C}$ bonding obtained from, molecular and periodic calculations, refinement on MSFs only and joint refinement. The periodic spin electron distribution around this bond is clearly different from the molecular one. As previously shown (see Fig. 6), the spin density around the two atoms is strongly affected by the nearest molecules. The spin electron distribution in the vicinity of the oxygen atom becomes completely negative. For the carbon atom, we found that the positive contours are broadened and spread along both directions of the Xaxis. Our results show that the corresponding spin density is improved by the MSFs only, but it remains in disagreement with the periodic results. By combining the MCPs with the MSFs, the resulting spin densities are in good agreement with periodic calculations: the refined spin densities on the oxygen atom are negative and consistent with the periodic one. However, to compare the resulting spin densities around the carbon and nitrogen atoms from molecular calculation or model refinements with the corresponding spin densities obtained 
FIG. 7: VB Spin density maps in the perpendicular plane to the urea molecule along the $\mathrm{X}$-axis direction. $\mathrm{U}$ is a vector collinear to the $\mathrm{O}=\mathrm{C}$ bond. Upper panel: Computed spin resolved electron densities for molecular (a) and periodic calculations (b). Second panel: Resulting spin density from Dzêta $(\mathbf{c})$ and Dzêta + Pop $(\mathbf{d})$ refinement from the MSFs only. Lower panel: The same as the second panel but from the joint refinement strategy (MSFs + MCPs). Contours at intervals of $\pm 0.01 \times 2^{n} \mu_{B} \AA^{-3}(\mathrm{n}=0-12)$ : positive and negative contours are, respectively, blue solid lines and red dashed lines, and neutral contours are green dashed lines.

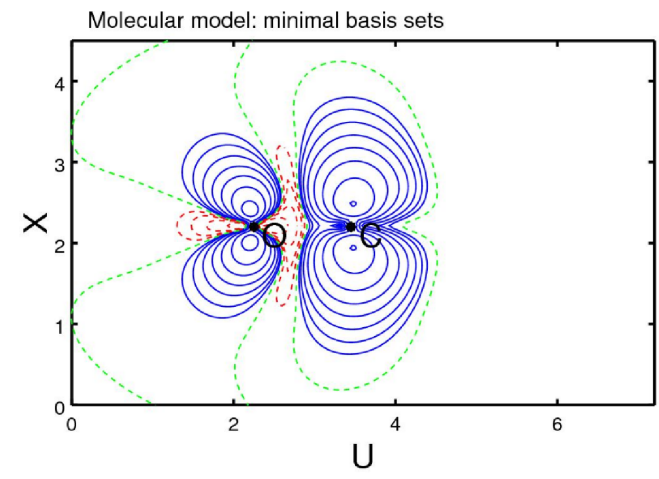

(a)

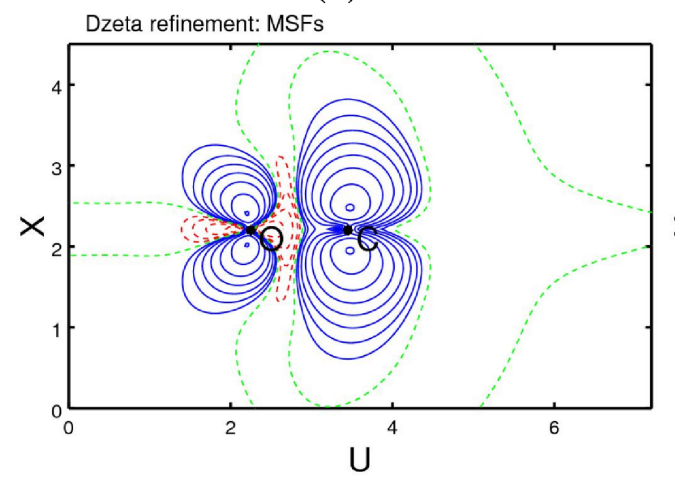

(c)

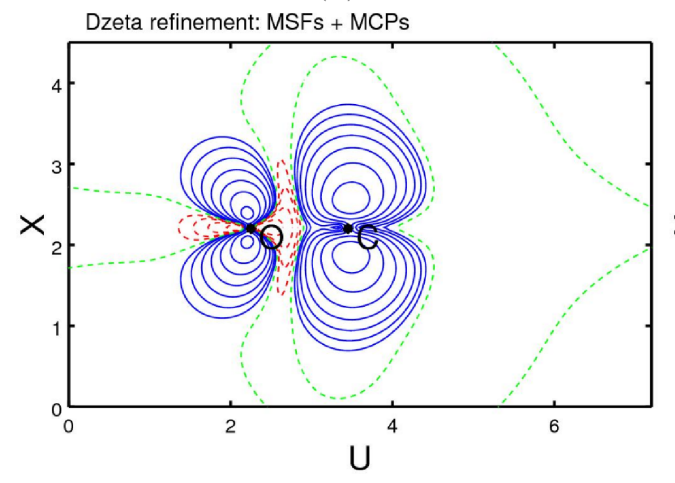

(e)

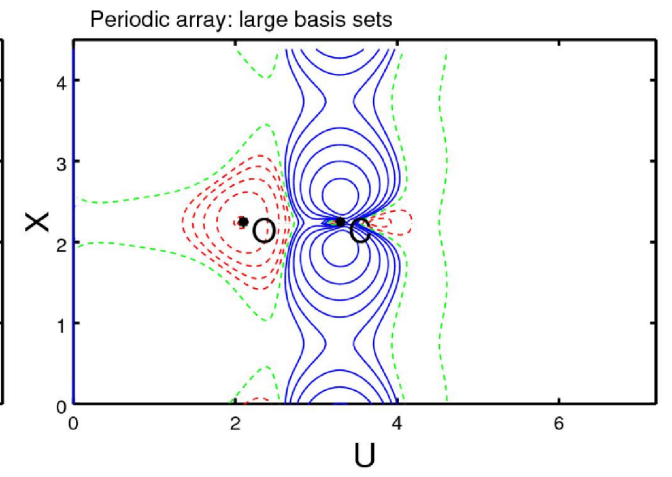

(b)

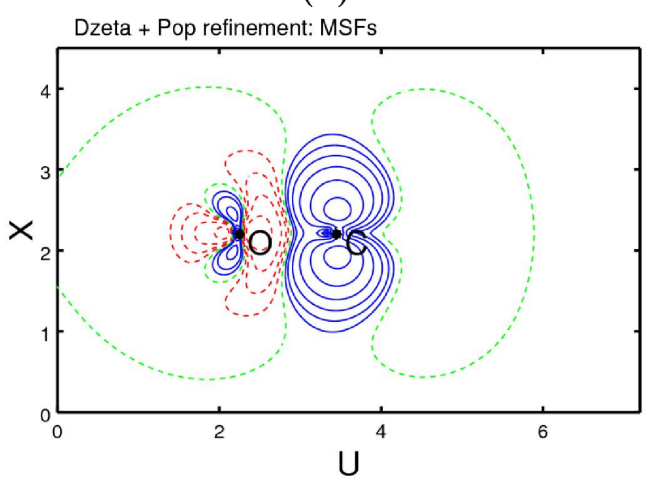

(d)

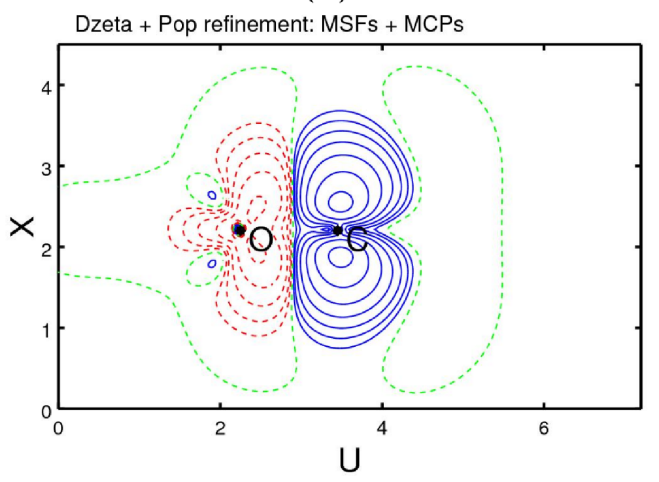

(f) 
FIG. 8: V B Spin density maps in the perpendicular plane to the urea molecule along the $\mathrm{X}$-axis direction. $\mathrm{U}$ is a vector collinear to the $\mathrm{C}-\mathrm{N}$ bond. Upper panel: Computed spin resolved electron densities for molecular (a) and periodic calculations (b). Second panel: Resulting spin density from Dzêta $(\mathbf{c})$ and Dzêta + Pop $(\mathbf{d})$ refinement with respect to the MSFs only. Lower panel: The same as the second panel but obtained by means of the joint refinement strategy (MSFs + MCPs). Contours at intervals of $\pm 0.01 \times 2^{n} \mu_{B} \AA^{-3}$ $(\mathrm{n}=0-12)$ : positive and negative contours are, respectively, blue solid lines and red dashed lines, and neutral contours are green dashed lines.

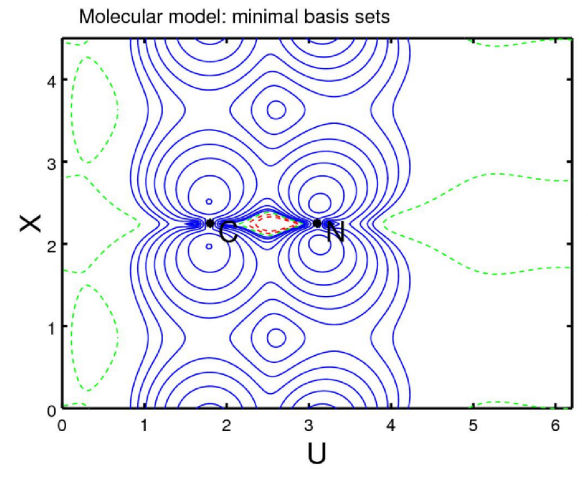

(a)

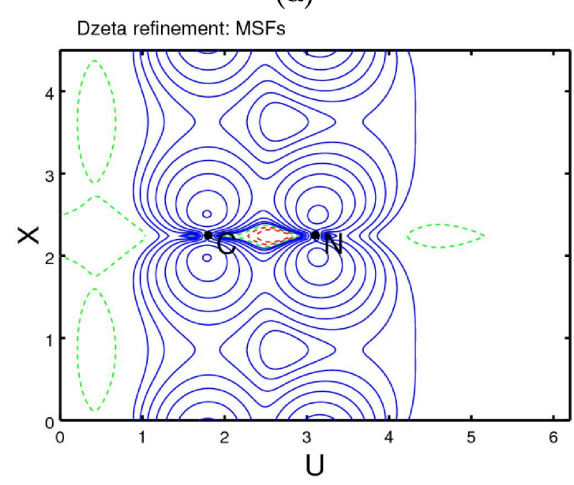

(c)

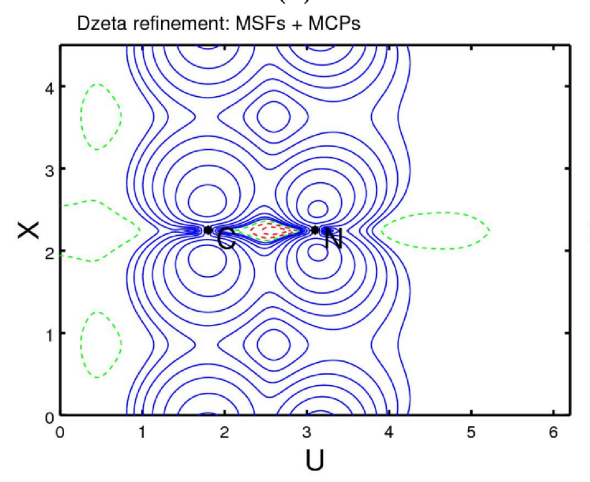

(e)

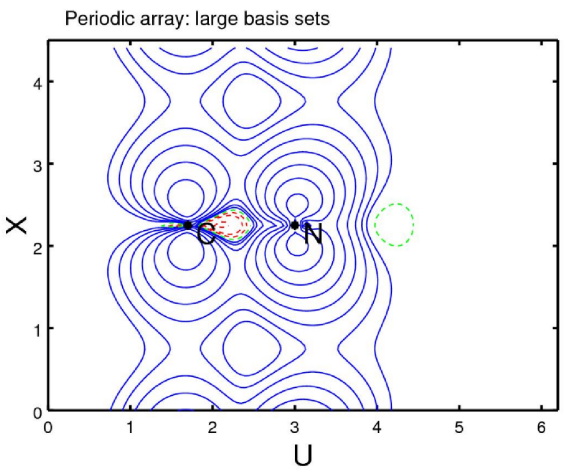

(b)

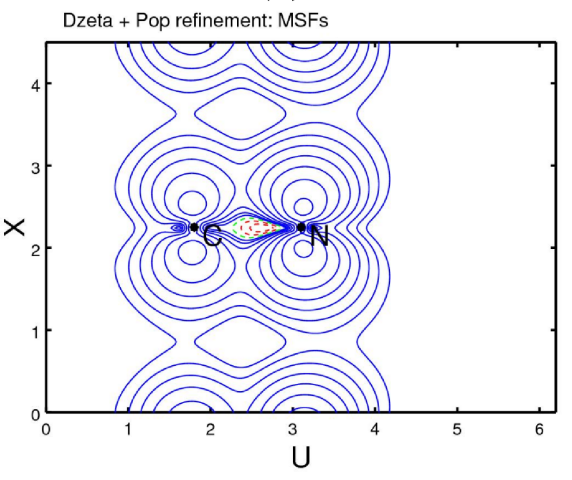

(d)

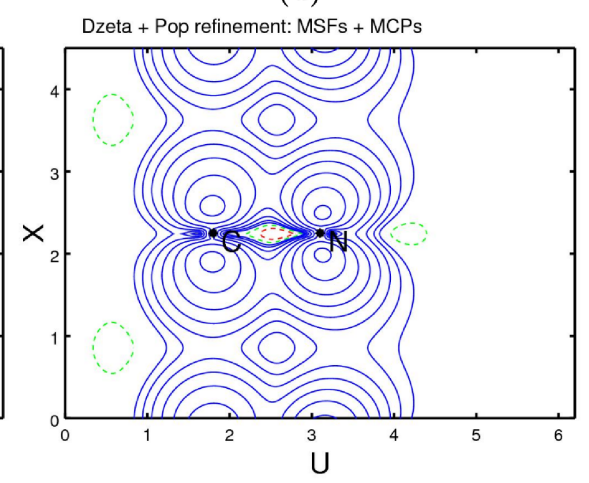

(f) 
from periodic calculations, the additional contributions of nearest neighbors are included as follows:

$$
\rho(\mathbf{r})=\rho(\mathbf{r}-\mathbf{R})+\rho(\mathbf{r})+\rho(\mathbf{r}+\mathbf{R}),
$$

where $\mathbf{R}=R \mathbf{u}_{X}, R=3 \AA$, the intermolecular distance between the urea molecules in the periodic array along the $\mathrm{X}$-axis. Fig. 8 shows the computed spin densities (upper panel) along the C-N bonds obtained from Eq. 28 for comparison with the resulting spin densities from refinement on MSFs only (middle panel) and from joint refinement (lower panel). The molecular spin density around the C-N bonds is changed by means of the MSFs, but still different from the periodic one. However, by combining the MCPs and the MSFs, the resulting spin density compares very well to the periodic spin density. We deduce therefore that the MCPs have a strong effect on the refinement of the spin density (as can clearly be seen on the diagonal regions of the 1-SRDM in Fig. 9).

Fig. 9 shows the $1-S R D M$ maps following the $\mathrm{O}=\mathrm{C}-\mathrm{N}-\mathrm{H}$ path obtained from molecular and periodic calculations, Dzêta and Dzêta + Pop refinement on MSFs only and from joint refinement. The differences between the molecular and the periodic calculations are also clearly seen on the off-diagonal regions of the 1-SRDM. The 1-SRDM are strongly affected by the neighboring molecules. It's surprising, that the refinement on MSFs only made a slight change on the off-diagonal regions of the molecular 1-SRDM (precisely in the vicinity of the $\Gamma^{(1)}\left(\mathbf{r}=1.6, \mathbf{r}^{\prime}=3.9\right)$ point $)$. However, as expected, the off-diagonal regions of the 1-SRDM are strongly affected by the MCPs (see lower panel of Fig. 9). The changes made by MCPs appear in the regions that describe the interactions between oxygen and carbon, oxygen and nitrogen, and carbon and nitrogen atoms. The 1-SRDM obtained from the proposed joint refinement strategy provides a very satisfactory qualitative agreement with the periodic calculation. The potential benefits brought by the joint refinement compared with the MSFs refinement are clearly illustrated in Fig. 10.

Fig. 10 shows a comparison of the computed (upper panel) 1-SRDM with the result from refinement on MSFs only (middle panel) and MSFs+MCPs (lower panel) for the same path of Fig. 9 at $0.5 \AA$ from the molecular plane along the $\mathrm{X}$-axis direction, respectively. The calculation shows that the periodic 1-SRDM is strongly affected by the nearest neighbors. It can be seen that the refinement on MSFs only and periodic calculations yield very different results. However, by combining MCPs with MSFs, the resulting 1-SRDM are in good 
FIG. 9: VB 1-SRDM $\Gamma^{(1)}\left(\mathbf{r}, \mathbf{r}^{\prime}\right)$ of the urea molecule is plotted along the $\mathrm{O}=\mathrm{C}-\mathrm{N}-\mathrm{H}$ direction (in the plan of the molecule). The upper panel shows the molecular (a) and periodic (b) computation of the 1-SRDM. The second panel shows the 1-SRDM after a Dzêta $(\mathbf{c})$ and Dzêta + Pop $(\mathbf{d})$ refinement relative to the MSFs only. The lower panel is the same as the second panel, but by means the joint refinement (MSFs + MCPs). Contours at intervals of $\pm 0.01 \times 2^{n} \mu_{B} \AA^{-3}(\mathrm{n}=0-20)$ : positive and negative contours are, respectively, blue solid lines and red dashed lines, and neutral contours are green dashed lines.

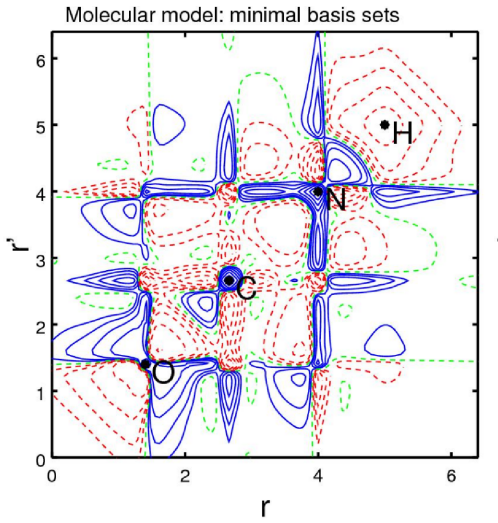

(a)

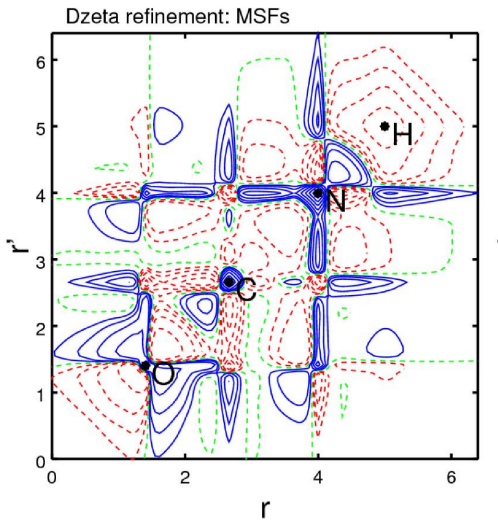

(c)

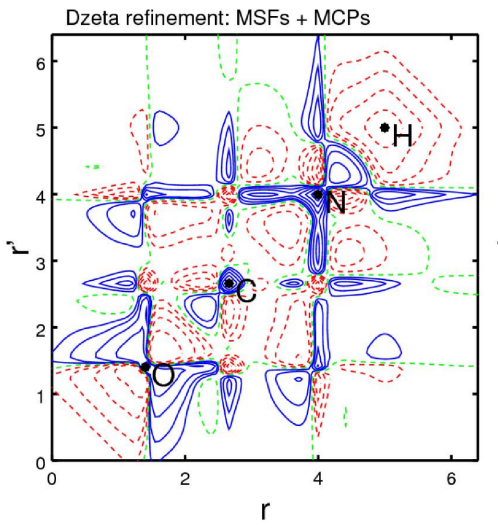

(e)

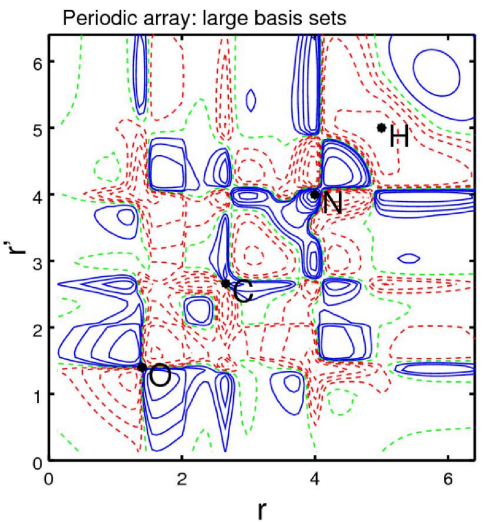

(b)

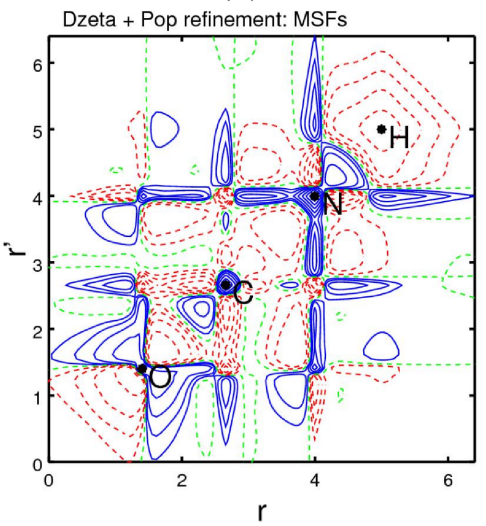

(d)

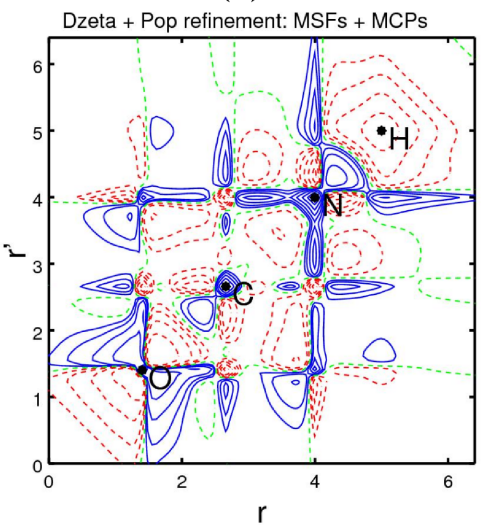

(f) 
FIG. 10: $\mathrm{VB}$ 1-SRDM $\Gamma^{(1)}\left(\mathbf{r}, \mathbf{r}^{\prime}\right)$ of the urea molecule is plotted along the $\mathrm{O}=\mathrm{C}-\mathrm{N}-\mathrm{H}$ direction (above the plane of the molecule by $0.5 \AA$ ). The upper panel shows the molecular (a) and periodic (b) computation of the 1-SRDM. The second panel shows the 1-SRDM after a Dzêta $(\mathbf{c})$ and Dzêta + Pop $(\mathbf{d})$ refinement relative to the MSFs only. The lower panel is the same as the second panel, but by means the joint refinement (MSFs and magnetic Compton profile). Contours at intervals of $\pm 0.01 \times 2^{n} \mu_{B} \AA^{-3}(\mathrm{n}=0-20)$ : positive and negative contours are, respectively, blue solid lines and red dashed lines, and neutral contours are green dashed lines.

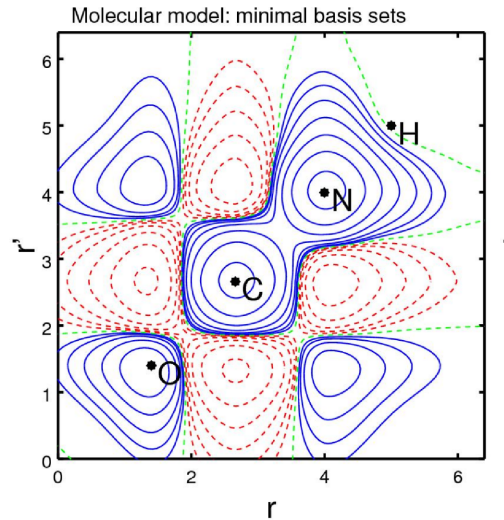

(a)

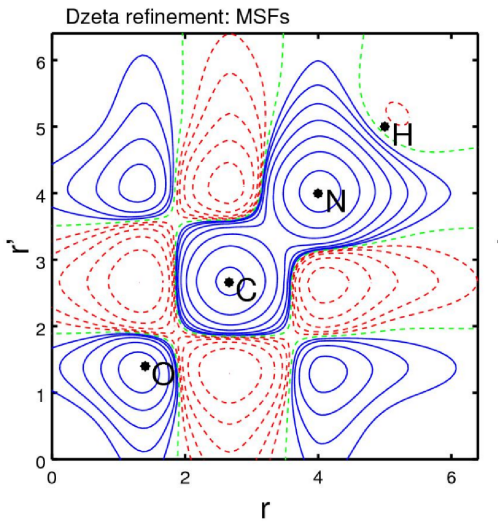

(c)

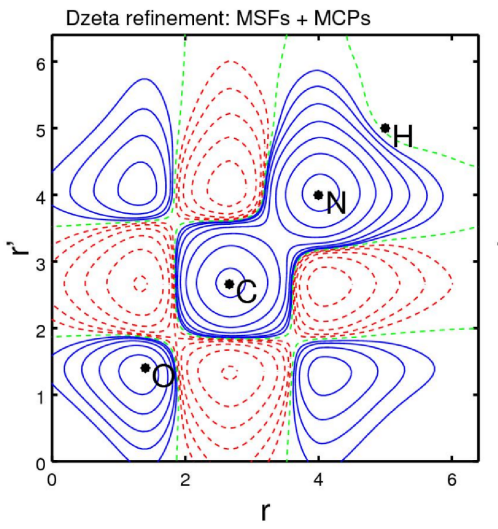

(e)

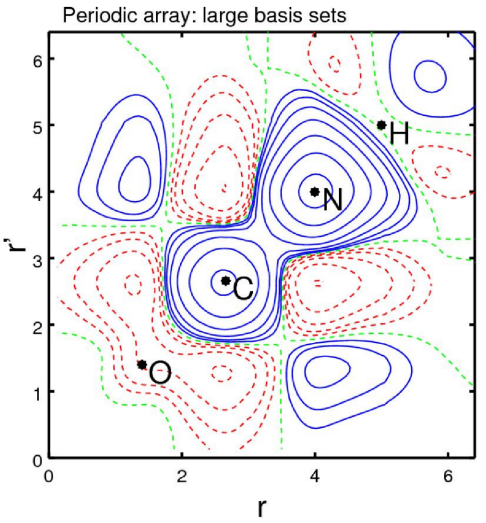

(b)

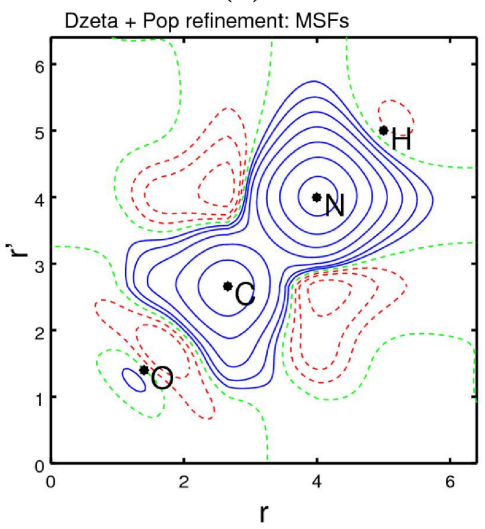

(d)

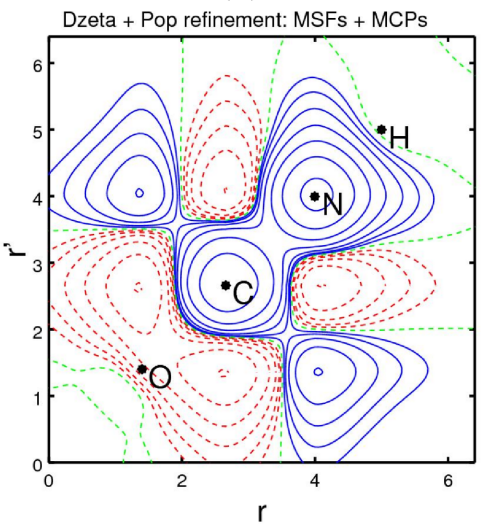

(f) 
FIG. 11: VB Computed MCPs for the following directions [ $\left[\begin{array}{lll}1 & 0 & 0\end{array}\right],\left[\begin{array}{lll}1 & 1 & 0\end{array}\right]$ and $\left[\begin{array}{lll}0 & 1 & 0\end{array}\right]$ as a function of the momentum q. For each direction, the resulting MCPs from periodic calculation (CRYS 14, red solid lines) is compared to resulting MCPs from, molecular calculation (G09, black solid lines), Dzêta refinement (Dzeta Ref, green solid lines) and Dzêta + Pop refinement (Dzeta+Pop Ref, blue dashed lines).
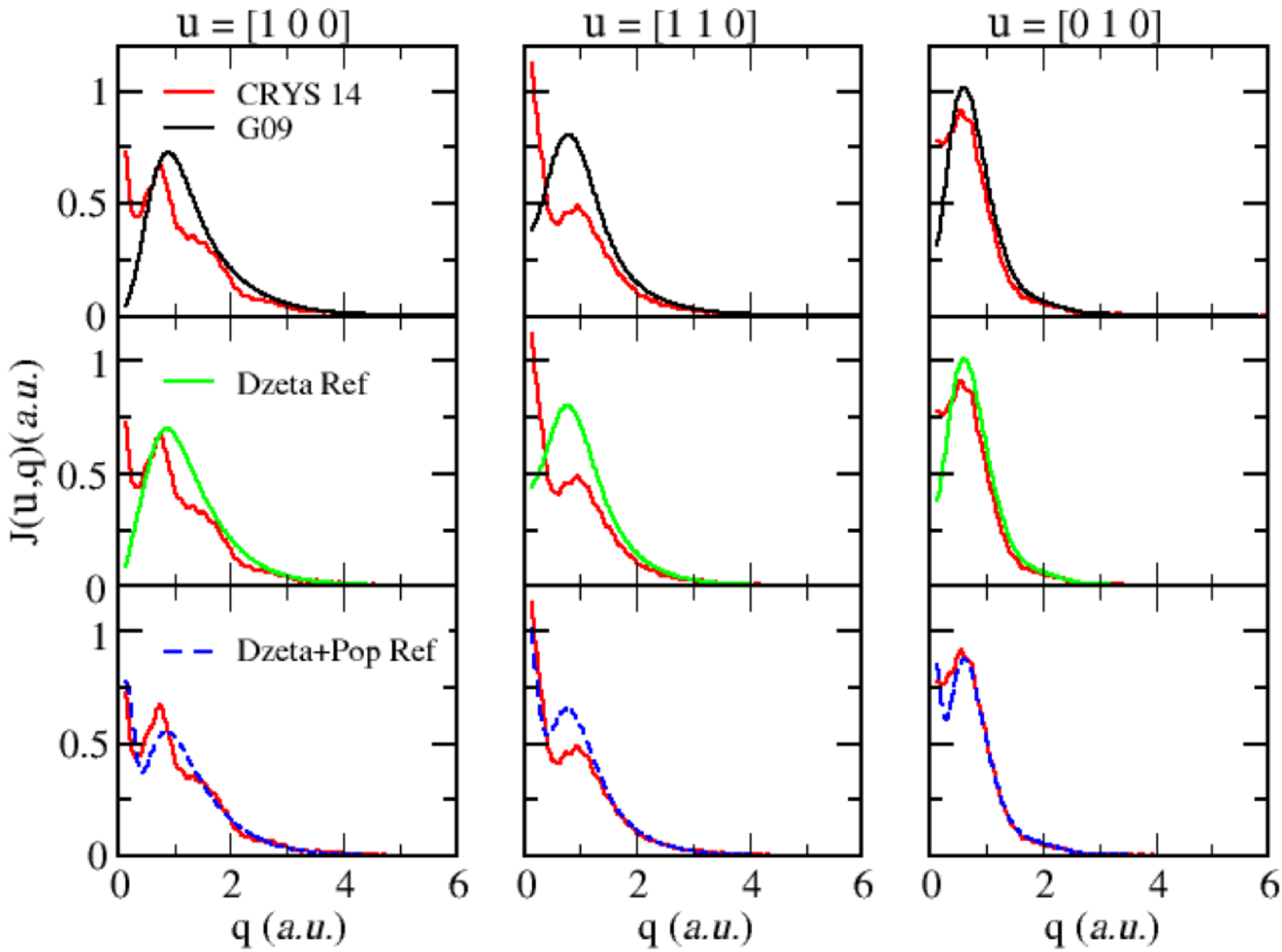

agreement with the periodic calculation. This emphasizes the essential role of the MCPs in the reconstruction the spin resolved density matrices.

We deduce that the joint refinement has a stronger effect on the $\pi$ electron distributions than on the $\sigma$ one. This is due to the fact that in our model we only adjust the occupation numbers of selected states close to the Fermi level, i.e. delocalized states. To obtain more accurate results for the $\sigma$ electron distribution, we should modify the occupation numbers for very localized states (much lower eigenvalues), which is not considered in this model.

Fig. 11 shows a comparison of the resulting MCPs from molecular calculation, Dzêta refinement and Dzêta + Pop refinement with the resulting MCPs from periodic calculation. As can be clearly seen, the molecular and the periodic calculations (see upper panel of Fig.11) 
are very different for all directions. This is due to the fact that the urea molecules are in interaction in the crystal phase, while isolated in the molecular gas phase. Our results show that the variation of the basis functions improves the MCPs, but it remains inconsistent with the periodic one. However, the variation of the spin population matrix together with the variation of the basis functions finally give very satisfactory results. The convergent MCPs from periodic calculation and joint refinement are shown to be consistent.

\section{CONCLUSIONS}

The paper describes our joint refinement model of the 1-SRDM for a molecular array using simultaneously different pseudo data sets (MSFs and MCPs). This model is implemented as post processing of the GAUSSIAN packages. To validate our model, we have performed two sets of calculations for a periodic array of urea with and without interaction between molecules using the CRYSTAL14 packages. These served as two sets of pseudo data for the refinement. For both molecular and periodic calculations, we have used local atomic orbitals as a basis set. To obtain 1-SRDM with good accuracy, we have modified (i) the basis functions by varying the atomic radial extensions and (ii) the spin population matrix by varying the occupation numbers for a selection of eigenstates, with respect to MSFs only and to both MSFs and MCPs. As expected, our results show that the MSFs improve the diagonal regions of the $1-S R D M$, but do not make it possible to describe very fine details of the spin distributions for all regions. We have shown that the joint refinement model gives more accurate results than the refinement on MSFs only. The joint refinement model

gives very satisfactory results compared with those obtained from periodic calculations of the periodic array of urea. We found that the MCPs play a central role in the refinement of the 1-SRDM, because it has a strong effect not only on the off-diagonal elements but also on its diagonal elements.

\section{ACKNOWLEDGMENTS}

This work was financed by the French National Research Agency (ANR) project MTMED (Multi Techniques Modeling of Electron Densities). We would like to acknowledge fruitful discussion with B. Gillon, N. Claiser, M. Souhassou, C. Lecomte and P. Cortona. This work 
was performed using HPC resources from Fusion computing mesocenter at CentraleSupelec Paris. We are greatly indebted to Julie McDonald for a careful reading of this paper.

1 T. L. Gilbert, Phys. Rev. B 12, 2111 (1975).

2 N. N. Lathiotakis and M. A. L. Marques, J. Chem. Phys. 128, 184103 (2008).

3 P. O. Löwdin, Phys. Rev. 97, 1474 (1955).

4 A. J. Coleman, Rev. Mod. Phys. 35, 668 (1963).

5 R. McWeeny, Rev. Mod. Phys. 32, 335 (1960).

6 E. R. Davidson, Reduced Density Matrices in Quantum Chemistry (1976).

7 J. M. Gillet and P. J. Becker, J. Phy. Chem. solids 65, 2017 (2004).

8 W. L. Luken, Int. J. Quant. Chem. 22, 889 (1982).

9 N. K. Hansen and P. Coppens, Acta Cryst., Sect. A 34, 909 (1978).

10 D. Jayatilaka and D. J. Grimwood, Acta Cryst., Sect. A 57, 76 (2001).

11 G. P. Barnett and H. Shull, Phys. Rev. 153, 61 (1967).

12 P. Krusius, H. Isomäki, and B. Kramer, Phys. Rev. B 19, 1818 (1979).

13 J. M. Gillet, C. Fluteaux, and P. J. Becker, Phys. Rev. B 60, 2345 (1999).

14 J. M. Gillet, P. J. Becker, and P. Cortona, Phys. Rev. B 63, 235115 (2001).

15 J. M. Gillet, Acta Cryst., Sect. A 63, 234 (2007).

16 I. A. Kibalin, Z. Yan, A. B. Voufack, S. Gueddida, B. Gillon, A. Gukasov, F. Porcher, A. M. Bataille, F. Morini, N. Claiser, M. Souhassou, C. Lecomte, J. M. Gillet, M. Ito, K. Suzuki, H. Sakurai, Y. Sakurai, C. M. Hoffmann, and X. P. Wang, Phys. Rev. B 96, 054426 (2017).

17 Z. Yan, I. A. Kibalin, N. Claiser, S. Gueddida, B. Gillon, A. Gukasov, A. B. Voufack, F. Morini, Y. Sakurai, M. Brancewicz, M. Itou, M. Itoh, N. Tsuji, M. Ito, M. Souhassou, C. Lecomte, P. Cartona, and J. M. Gillet, Phys. Rev. B 96, 054427 (2017).

18 M. Deutsch, N. Claiser, S. Pillet, Y. Chumakov, P. Becker, J. M. Gillet, B. Gillon, C. Lecomte, and M. souhassou, Acta Cryst., Sect. A 68, 675 (2012)

19 M. Deutsch, B. Gillon, N. Claiser, J. M. Gillet, C. Lecomte, and M. Souhassou, IUCrJ 1, 194 (2014).

20 P. J. Brown, J. B. Forsyth, and R. Mason, Philos. Trans. R. Soc. B 290, 481 (1980). 
21 P. Coppens, X-ray Charge Densities and Chemical Bonding: International Unionof Crystallography Texts on Crystallography, No4 (Oxford University Press, 1997).

22 W. Weyrich, One-electron density matrices and related observables in: C. Pisani (Ed.), Quantum Mechanical Ab-initio Calculation of the Properties of Crystalline Materials (Spinger Berlin pp. 245-272, 1996).

23 M. J. Cooper, P. E. Mijnarends, N. Shiotani, N. Sakai, and A. Bansil, X-ray Compton scattering (Oxford University Press, 2004).

24 J. Schweizer, Neutron Scattering from Magnetic Materials, edited by T. Chatterji, Ch. 4. (Amesterdam: Elsevier, 2006).

25 S. Pillet, M. Souhassou, Y. Pontillon, A. Caneschi, D. Gatteschi, and C. Lecomte, New J. Chem. 25, 131 (2001).

26 F. James, MINUIT Function Minimization and Error Analysis: Reference Manual Version 94.1 (1994).

27 A. Shukla, E. D. Issaacs, D. R. Hamann, and P. M. Platzman, Phys. Rev. B 64, 052101 (2001).

28 R. Dovesi, M. Causa, R. Orlando, C. Roetti, and V. R. Saunders, J. Chem. Phys. 92, 7402 (1990).

29 Z. Lin, Z. Wang, C. Chen, and M. H. Lee, J. Chem. Phy. 118, 2349 (2003).

30 C. Pisani, Quantum-Mechanical Ab-initio Calculation of the Properties of Crystalline Materials (1996).

31 R. Dovesi, R. Orlando, A. Erba, C. M. Zicovich-Wilson, B. Civalleri, S. Casassa, L. Maschio, M. Ferrabone, M. D. la Pierre, P. D’Arco, , Y. Noël, M. Causá, M. Rérat, and B. Kirtman, Int. J. Quantum Chem. 114, 1287 (2014).

32 R. Dovesi, V. R. Saunders, C. Roetti, R. Orlando, C. M. Zicovich-Wilson, F. Pascale, B. Civalleri, K. Doll, N. M. Harrison, I. J. Bush, P. D’Arco, M. Llunell, M. Causa, and Y. Noel, CRYSTAL14 User's Manual, University of Torino, Torino (2014).

33 M. F. Peintinger, D. V. Oliveira, and T. Bredow, J. Comput. Chem. 34, 451 (2013).

34 M. J. Frisch, G. W. Trucks, H. B. Schlegel, G. E. Scuseria, M. A. Robb, J. R. Cheeseman, G. Scalmani, V. Barone, B. Mennucci, G. A. Petersson, H. Nakatsuji, M. Caricato, X. Li, H. P. Hratchian, A. F. Izmaylov, J. Bloino, G. Zheng, J. L. Sonnenberg, M. Hada, M. Ehara, K. Toyota, R. Fukuda, J. Hasegawa, M. Ishida, T. Nakajima, Y. Honda, O. Kitao, H. Nakai, T. Vreven, J. A. Montgomery, J. E. Peralta, F. Ogliaro, M. Bearpark, J. J. Heyd, E. Brothers, 
K. N. Kudin, V. N. Staroverov, R. Kobayashi, J. Tomasi, M. Cossi, N. Rega, J. M. Millam, M. Klene, J. E. Knox, J. B. Cross, V. Bakken, C. Adamo, J. Jaramillo, R. Gomperts, R. E. Stratmann, O. Yazyev, A. J. Austin, R. Cammi, C. Pomelli, J. W. Ochterski, R. L. Martin, K. Morokuma, V. G. Zakrzewski, G. A. Voth, P. Salvador, J. J. Dannenberg, S. Dapprich, A. D. Daniels, O. Farkas, J. B. Foresman, J. V. Ortiz, J. Cioslowski, and D. J. Fox, Gaussian 09 (Revision B.01) (2009).

35 M. S. Gordon, J. S. Binkley, J. A. Pople, W. J. Pietro, and W. J. Hehre, J. Am. Chem. Soc. 104, 2797 (1982).

36 J. S. Binkley, J. A. Pople, and W. J. Hehre, J. Am. Chem. Soc. 102, 939 (1980).

37 W. J. Pietro, M. M. Francl, W. J. Hehre, D. J. DeFrees, J. A. Pople, and J. S. Binkley, J. Am. Chem. Soc. 104, 5039 (1982).

38 K. D. Dobbs and W. J. Hehre, J. Comput. Chem. 7, 359 (1986).

39 K. D. Dobbs and W. J. Hehre, J. Comput. Chem. 8, 861 (1987)

40 K. D. Dobbs and W. J. Hehre, J. Comput. Chem. 8, 880 (1987).

41 M. J. Frisch, J. A. Pople, and J. S. Binkley, J. Chem. Phys. 80, 3265 (1984).

42 T. Clark, J. Chandrasekhar, G. W. Spitznagel, and P. v. R. Schleyer, J. Comput. Chem. 4, 294 (1983).

43 M. W. Schmidt, K. K. Baldridge, J. A. Boatz, S. T. Elbert, M. S. Gordon, J. H. Jensen, S. Koseki, N. Matsunaga, K. A. Nguyen, S. Su, T. L. Windus, M. Dupuis, and J. A. Montgomery, J. Comput. Chem. 14, 1347 (1993). 